\title{
Morphology and Orientation of Crystallites of Simultaneous Biaxially Stretching Ultra-High Molecular Weight Polyethylene Films Prepared by Gelation/Crystallization from Solutions
}

\author{
Teruo NaKashima, Chunye Xu, ${ }^{*}$ Yuezhen Bin, ${ }^{*}$ and Masaru Matsuo ${ }^{* \dagger}$ \\ Kinki University of Toyooka Junior College, Toyooka 668-8580, Japan \\ * Department of Textile and Apparel Science, Faculty of Human \\ Life and Environment, Nara Women's University, Nara 630-8263, Japan
}

(Received June 22, 2000; Accepted September 26, 2000)

\begin{abstract}
Simultaneous biaxially stretching was carried out using ultra-high molecular weight polyethylene dry gel films which were prepared by crystallization from solutions. The concentrations were $0.45,0.5,0.6,0.7,0.8,0.9$, and $1.0 \mathrm{~g} / 100 \mathrm{~mL}$. The maximum draw ratio was dependent upon the concentration of the solutions. The greatest significant drawability could be realized at $0.9 \mathrm{~g} / 100 \mathrm{~mL}$ which is much higher than the optimum concentration of $0.45 \mathrm{~g} / 100 \mathrm{~mL}$ assuring the draw ratio $>300$-fold for uniaxially stretching. Young's modulus of the biaxially stretched film was much lower than that of uniaxial films with the same draw ratio (70-fold), although the Young's modulus of the film with the maximum draw ratio was much higher than that of commercial films. To address this problem, the orientation function of crystallites was determined from the orientation of the reciprocal lattice vectors of the crystal planes. As the result, it turned out that the $c$ - and $a$-axes are oriented predominantly to the film surface but the orientational degree of the $c$ axis is not remarkable in spite of high draw ratio of $8.7 \times 8.7$. The second order orientation factor was estimated from birefringence by subtracting the crystalline contribution from the total birefringence. These results indicated that the preferential orientation of the $c$-axis to the stretching direction is mainly due to the rotation of crystallites around their $b$-axis leading to straining of tie molecules. Furthermore, the ultimate value of Young's modulus was estimated by assuming an ideal simultaneous biaxially stretching film with 100\% crystallinity and the perfect orientation of the $c$-axis parallel to the film surface. Even so, the predicted value was less than $10 \mathrm{GPa}$, when the elastic compliance of a crystal unit by Odajima and Zehnder were employed. This indicates the difficulty in producing high modulus and high strength polyethylene sheets in terms of theoretical aspects.
\end{abstract}

KEY WORDS Simultaneous Biaxially Stretching / Ultra-High Molecular Weight Polyethylene / Dry Gel Films / Maximum Draw Ratio / Orientation Function of Crystallites / Ultimate Value of Young's Modulus /

Gel deformation is well known as an excellent method to prepare high-modulus and high-strength polyethylene fibers. This method was first proposed by Smith, Lemstra, Kalb, and Pennings ${ }^{1}$ and was mainly developed by Smith and Lemstra. ${ }^{2-5}$ They demonstrated that the effective drawability of ultra-high molecular weight polyethylene (UHMWPE) made by the gel deformation method is dramatically enhanced using specimens spun or cast from semi-dilute solutions to form macroscopic gels. It turns out that the maximum achievable draw ratio for UHMWPE depends principally on the concentration of solution from which the gel is made. ${ }^{5,6}$ Namely, the dry gel films of UHMWPE (molecular weight of $6 \times$ $10^{6}$ ) could be elongated up to 400 times and the Young's modulus reached $216 \mathrm{GPa}^{7}$, when the gels were prepared from solutions with the optimum concentration. The value is close to the ultimate value corresponding to the crystal lattice modulus $(220-235 \mathrm{GPa})$ along the chain direction measured by X-Ray diffraction at room temperature. ${ }^{7,8}$ Incidentally, the crystal lattice modulus is reported to be $254 \mathrm{GPa}^{9}$ at $-155^{\circ} \mathrm{C}$ close to the glass transition temperature. This means that the crystal lattice modulus increases when the local motion of chain segments is frozen.

With regard to biaxially stretching, a number of papers for the deformation mechanism of poly(ethylene terephthalate), ${ }^{10}$ polypropylene ${ }^{11}$ and poly(vinyl alcohol)

${ }^{\dagger}$ To whom all correspondence should be addressed. films ${ }^{12}$ have been published by several authors. They studied molecular orientation by X-Ray diffraction and birefringence quantitatively as well as the deformation of superstructure such as spherulites by polarized microscopy. As a further interesting problem, biaxially stretching of UHMWPE dry gel films with significant greatest drawability under uniaxially stretching also causes a significant effect on the biaxially stretching. The biaxially drawing for UHMWPE was first done by Miyasaka et $a l .^{13-15}$ They studied the development of fibrillar texture and mechanical properties of UHMWPE dry gel films. Further considerations of the deformation mechanism and mechanical properties have been published by Gerrits et $a l .{ }^{16-18}$ and Bastiannsen et al. ${ }^{19}$ in terms of experimental and theoretical aspects. They pointed out that the Young's modulus and tensile strength are not improved in contrast to those of uniaxially drawn tapes. A detailed analysis of the deformation mechanism was discussed in terms of crystal plasticity on the basis of the planar orientation of the crystal planes. Among their reports, a detailed analysis was done by Gerrits and Young. ${ }^{18}$ According to their report, ${ }^{18}$ crystal deformation proceed both by slip on the (100) and (110) planes, resulting in a (100) texture in a similar way to crystal deformation in uniaxially drawn polyethylene and by (110) (110) transverse slip and/or (310) twinning which results in a (110) texture. However, their analysis is somewhat simpler than other treatments of the morphology and the orientation of crystal- 
lites of uniaxially stretched films. ${ }^{20,21}$

To give a more quantitative treatment of simultaneous biaxially stretching mechanism of UHMWPE dry gel films, this paper deals with the morphology and mechanical properties of the films in relation to the optimum concentration assuring the greatest significant drawability of UHMWPE. In doing so, the deformation mechanism was analyzed using the orientation distribution function of crystallites. The function can be determined from the uniaxial orientation distribution functions of the reciprocal lattice vectors of the crystal planes around the film normal direction by using the method of Roe and Krigbaum. ${ }^{22-24}$ Furthermore, an ultimate value of Young's modulus of simultaneous biaxially stretched film was postulated by using the relationship of elastic compliance between bulk and crystal unit in an ideal case with $100 \%$ crystallinity and perfect orientation of the $c$-axes parallel to the film surface.

\section{EXPERIMENTAL}

\section{Sample Preparation}

The samples used in this experiment were UHMWPE (Hercules 1900/90189) with a viscosity-average molecular weight $\left(\bar{M}_{\mathrm{v}}\right)$ of $6 \times 10^{6}$. The solvent was decalin. The concentrations of UHMWPE chosen were 0.4, 0.6, 0.7, $0.8,0.9$, and $1.0 \mathrm{~g} / 100 \mathrm{~mL}$. Decalin solutions were prepared by heating the well-blended polymer-solvent mixture at $135^{\circ} \mathrm{C}$ for $40 \mathrm{~min}$ under nitrogen. The solution was stabilized with $3 \% \mathrm{w} / \mathrm{w}$ of antioxidant (di-t-butyl- $p$ cresol) against UHMWPE. The hot homogenized solution was quenched to room temperature by pouring it into an aluminum tray, thus generating a gel. The decalin was allowed to evaporate from the gels under ambient conditions. The resulting dry gel film was vacuumdried for 1 day to remove residual trace of decalin.

The dry gel film was cut into strips of $90 \times 90 \mathrm{~mm}$. The specimens were held at $150^{\circ} \mathrm{C}$ for $5 \mathrm{~min}$ and elongated biaxially to the desired ratio using an Iwamoto biaxial stretcher. The draw ratio was determined in the usual way by measuring the displacement of ink marks placed $5 \times 5 \mathrm{~mm}$ apart on the specimen prior to drawing. Immediately after stretching, the sample fixed in the stretcher was quenched to room temperature. Unlike the case of uniaxial elongation, no necking occurred under simultaneous biaxially stretching.

\section{Sample Characterization}

Table I summarizes the concentration dependence of the maximum draw ratio $\left(\lambda_{\max }\right)$ of dry gel films under biaxially and uniaxially stretching. For example, the draw ratio along two directions under simultaneous biaxially stretching is represented as $2 \times 2$. The attainable draw ratio increased with increasing concentration and the maximum draw ratio reached $8.7 \times 8.7$ for dry gel films prepared from solutions with $0.9 \mathrm{~g} / 100 \mathrm{~mL}$ concentration. The thickness of the film was less than $10 \mu \mathrm{m}$, which is thinner than that of the commercial films. All specimens except an undrawn film were transparent. In contrast, for uniaxially stretching, the draw ratio decreased with increasing concentration. The draw ratio reached 400 -fold at $0.45 \mathrm{~g} / 100 \mathrm{~mL}$, which corresponds to the optimum concentration assuring the greatest signifi-
Table I. Concentration dependence of the maximum draw ratio $\left(\lambda_{\max }\right)$ of the dry gel films under biaxial and uniaxial stretching

\begin{tabular}{ccc}
\hline Concentration $(\mathrm{g} / 100 \mathrm{~mL})$ & $\lambda_{\max }$ (biaxial) & $\lambda_{\max }($ uniaxial $)$ \\
\hline 0.45 & $4.0 \times 4.0$ & 400 \\
0.60 & $6.0 \times 6.0$ & 250 \\
0.70 & $7.3 \times 7.3$ & 230 \\
0.90 & $8.7 \times 8.7$ & 210 \\
1.0 & $7.8 \times 7.8$ & 19 \\
\hline
\end{tabular}

Table II. Draw ratio dependence of crystallinity and melting points of the films prepared from solutions with the indicated concentrations

\begin{tabular}{|c|c|c|c|}
\hline Concentration & \multirow{2}{*}{ Draw ratio } & Crystallinity & Melting point \\
\hline $\mathrm{g} / 100 \mathrm{~mL}$ & & $\%$ & ${ }^{\circ} \mathrm{C}$ \\
\hline 0.45 & $1.0 \times 1.0$ & 84.9 & 143 \\
\hline & $1.5 \times 1.5$ & 51.8 & 136 \\
\hline & $2.0 \times 2.0$ & 53.2 & 134 \\
\hline & $3.0 \times 3.0$ & 56.8 & 132 \\
\hline 0.6 & $1.0 \times 1.0$ & 85.7 & 143 \\
\hline & $2.0 \times 2.0$ & 56.1 & 140 \\
\hline & $6.0 \times 6.0$ & 63.2 & 138 \\
\hline 0.7 & $1.0 \times 1.0$ & 84.9 & 144 \\
\hline & $2.0 \times 2.0$ & 56.7 & 143 \\
\hline & $6.0 \times 6.0$ & 63.5 & 141 \\
\hline & $7.3 \times 7.3$ & 65.3 & 139 \\
\hline 0.8 & $1.0 \times 1.0$ & 86.8 & 144 \\
\hline & $2.0 \times 2.0$ & 57.4 & 143 \\
\hline & $6.0 \times 6.0$ & 63.7 & 141 \\
\hline & $7.7 \times 7.7$ & 65.6 & 140 \\
\hline 0.9 & $1.0 \times 1.0$ & 85.2 & 144 \\
\hline & $2.0 \times 2.0$ & 57.6 & 143 \\
\hline & $6.0 \times 6.0$ & 64.0 & 141 \\
\hline & $8.7 \times 8.7$ & 67.5 & 141 \\
\hline 1.0 & $1.0 \times 1.0$ & 84.9 & 144 \\
\hline & $2.0 \times 2.0$ & 58.3 & 143 \\
\hline & $6.0 \times 6.0$ & 65.2 & 141 \\
\hline & $7.8 \times 7.8$ & 70.1 & 141 \\
\hline
\end{tabular}

cant drawability.

The density of the films was measured by pycnometer with chlorobenzene-toluene as the medium. Since the density was very dependent on the presence of residual solvent in the film, great care was taken to remove the solvent. Samples were cut into fragments and subsequently vacuum-dried for 1 day prior to measuring the density. The dried fragments were immersed in chlorobenzene-toluene for a day to promote the penetration of the solvents into voids and then the density measurements were carried out. Crystallinity was calculated assuming intrinsic densities of crystalline and amorphous phases to be 1.000 and $0.852 \mathrm{~g} \mathrm{~cm}^{-3}$ respectively. ${ }^{25}$

The thermal behavior was estimated in terms of melting endotherms of differential calorimetry (DSC) curves. Dry gels, with $5 \mathrm{mg}$ weight, were placed in a standard aluminum sample pan. Samples were heated at a constant rate of $10^{\circ} \mathrm{C} \mathrm{min.}{ }^{-1}$

Table II summarizes change in melting point and crystyallinity as a function of draw ratio of the dry gel films prepared from solutions with the indicated concentrations. Upon the initial draw ratio from $1.0 \times 1.0$ (undrawn) to $2.0 \times 2.0$, crystallinity and melting point decrease indicating the crystal transformation process 


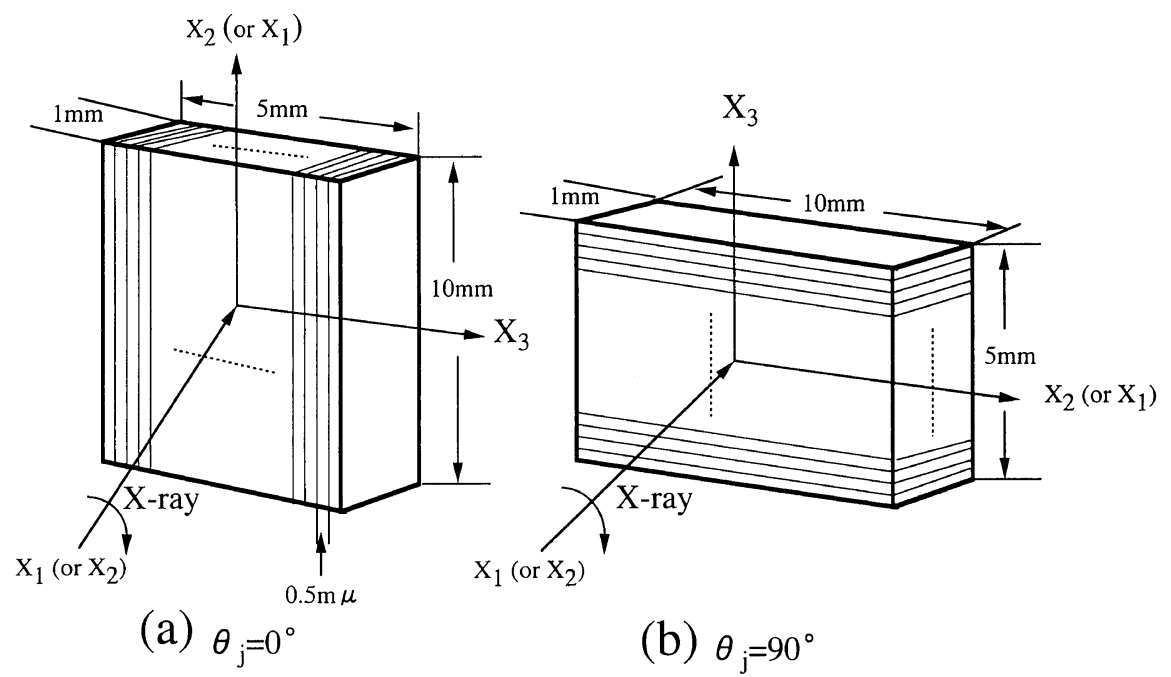

Figure 1. A number of thin stacked films to measure X-Ray diffraction intensity distribution as a function of twice the Bragg angle at (a) $\theta_{j}=0^{\circ}(\mathrm{b}) \theta_{j}=90^{\circ}$.

from a folded to a fibrous type. This tendency is most significant at the $0.45 \mathrm{~g} / 100 \mathrm{~mL}$ concentration. With further increase in draw ratio, crystallinity increased for all the specimens. The maximum crystallinity is $70.1 \%$, which is much lower than the crystallinity (90\%) of uniaxially stretched films with a draw ratio of 50 -fold. Here it is of interest to consider that in spite of an increase in crystallinity with increasing draw ratio $>2.0 \times 2.0$, the corresponding melting point decreased with increasing draw ratio. This tendency is less pronounced as the concentration increased. For example, the decreasing melting point of the specimens prepared from concentrations $>0.9 \mathrm{~g} / 100 \mathrm{~mL}$ is only $3^{\circ} \mathrm{C}$. Anyway, these values are much lower than the apparent melting point $\left(155.5^{\circ} \mathrm{C}\right)$ of the uniaxial elongation film with a draw ratio of 50 fold. ${ }^{26}$ Incidentally, the crystallinities estimated by density are in fairly good agreement with those measured by DSC.

\section{Estimation Method of Orientation Distribution Function by X-Ray Diffraction}

The X-Ray measurements were carried out with a 12 $\mathrm{kW}$ rotating-anode X-Ray generator (Rigaku RDA-rA) operated at $200 \mathrm{~mA}$ and $40 \mathrm{kV}$. Here we must emphasize that the information concerning crystal orientation around the film normal direction cannot be obtained by using usual X-Ray diffraction for the present specimens because of very thin films $(<5 \mu \mathrm{m})$. Therefore, no orientation function has ever been reported even for the reciprocal lattice vectors of crystal planes with very strong diffraction intensity such as the (110) and (200) planes for UHMWPE biaxially stretched films. We developed a small but refined instrument to stack a number of thin films as shown in Figure 1. In such a stacked condition, measurements of the X-Ray diffraction intensity could be performed using a horizontal scanning type goniometer, operating at a fixed time step scan of $0.1 / 40 \mathrm{~s}$ over a range of twice the Bragg angle $2 \theta_{\mathrm{B}}$ from 15 to $60^{\circ}$ and from 70 to $79^{\circ}$. The intensity distribution was measured as a function of a given rotational angle $\theta_{j}$ by rotating about the stretching direction at $2-5^{\circ}$ intervals from 0 to $90^{\circ} . \theta_{j}$ is $0^{\circ}$ and $90^{\circ}$ in Figure $1 \mathrm{a}$ and $1 \mathrm{~b}$, respectively.
Corrections of X-Ray diffraction intensity were made for air scattering, background noise, polarization, absorption and amorphous contribution. The intensity curve thus obtained was assumed to be due to the contribution of the intensity from the crystalline phase. The intensity curve $I_{\text {cry }}\left(2 \theta_{\mathrm{B}}\right)$ was separated into the contribution from the individual crystal planes, assuming that each peak had a symmetric form given by a Lorenzian function of in eq 1 , where $I_{j}^{\circ}$ is the maximum intensity of the $j$-th peak.

$$
I_{\text {cry }}\left(2 \theta_{\mathrm{B}}\right)=\sum_{j} \frac{I_{j}^{\mathrm{o}}}{1+\left(2 \theta_{\mathrm{o}}^{j}-2 \theta_{\mathrm{B}}\right)^{2} / \beta_{j}^{2}}
$$

Here $\beta_{j}$ is the half-width of the $j$-th peak at half of the peak intensity and $\theta_{o}^{j}$ is the Bragg angle at which the maximum intensity of the $j$-th peak appears. Using the same process at a given $\theta_{j}$ in the range from 0 to $90^{\circ}$, the intensity distribution $I_{j}\left(2 \theta_{\mathrm{B}}\right)$ can be determined for the respective $j$-th plane after integrating $I_{\text {cry }}\left(2 \theta_{\mathrm{B}}\right)$ by $2 \theta_{\mathrm{B}}$ at each $\theta_{j}$, and consequently the orientation distribution function $2 \pi q_{j}\left(\cos \theta_{j}\right)$ of the $j$-th reciprocal lattice vector may be given by

$$
2 \pi q_{j}\left(\cos \theta_{j}\right)=\frac{I_{j}\left(\theta_{j}\right)}{\int_{0}^{\pi / 2} I_{j}\left(\cos \theta_{j}\right) \sin \theta_{j} \mathrm{~d} \theta_{j}}
$$

The orientation distribution function of crystallites can be calculated on the basis of the orientation functions of the reciprocal lattice vectors measured by X-Ray diffraction, according to the method proposed by Roe and Krigbaum. $^{22-24}$

\section{Experimental Procedures}

Mechanical properties. To measure Young's modulus and tensile strength, specimens were elongated at $20^{\circ} \mathrm{C}$ with an Instron tester at a cross-head speed of $5 \mathrm{~mm}$ min. ${ }^{-1}$ The initial dimensions of the samples were length $20 \mathrm{~mm}$ and width $4 \mathrm{~mm}$. 
The complex dynamic tensile modulus was measured at $10 \mathrm{~Hz}$ over the temperature range from -150 to 170 ${ }^{\circ} \mathrm{C}$ by using a visco-elastic spectrometer (VES-F) obtained from Iwamoto Machine Co. Ltd. The length of the specimen between the jaws was $40 \mathrm{~mm}$ and the width was about $1.5 \mathrm{~mm}$. During measurement, the film was subjected to a static tensile strain in order to place the sample in tension during the axial sinusoidal oscillation, which had a peak deformation of $0.05-0.1 \%$. The complex dynamic modulus was measured by imposing a small dynamic strain to assure linear visco-elastic behavior of the specimen. ${ }^{27}$

Solid State ${ }^{13}$ C Nuclear Magnetic Resonance (NMR).

The ${ }^{13} \mathrm{C}$ CP/MAS (cross-polarization) and PST/MAS (pulse saturation) NMR measurements were carried out for dry gel films at room temperature with a JEOL, JMEX 270 spectrometer, operating at a static magnetic field of $6.34 \mathrm{~T}$. A radio frequency of $67.5 \mathrm{MHz}$ was used for detection of ${ }^{13} \mathrm{C}$ resonance. The magic-angle spinning rate was $5-5.5 \mathrm{kHz}$. The contact time in ${ }^{13} \mathrm{C}$ CP-MAS measurement was $2 \mathrm{~ms}$. The chemical shifts were mainly determined from the higher field signal (29.5 ppm) of adamantane relative to tetramethylsilane (TMS).

Other Characterizations. Measurements of scanning electron microscopy (SEM), wide angle X-Ray diffraction (WAXD) and small angle X-Ray scattering (SAXS) are described elsewhere in detail. ${ }^{28}$

\section{RESULTS AND DISCUSSIONS}

\section{Mechanical Properties}

Table III(a) shows Young's modulus and tensile strength at the maximum draw ratio of the films prepared by gelation/crystallization from solutions with the indicated concentrations $>0.7 \mathrm{~g} / 100 \mathrm{~mL}$. With increasing concentration, the values become higher and the maximum value occurred at $1.0 \mathrm{~g} / 100 \mathrm{~mL}$. Interestingly, the Young's modulus and tensile strength of the films $(7.8 \times 7.8)$ prepared from a $1.0 \mathrm{~g} / 100 \mathrm{~mL}$ solution are higher than those of the film $(8.7 \times 8.7)$ prepared from a $0.9 \mathrm{~g} / 100 \mathrm{~mL}$ solution. This indicates that Young's modulus is sensitive to the number of entanglements within the specimen, when the orientation of the $c$-axes increases to a certain degree. Namely, Young's modulus increases with increasing the number of entanglements. Table III(b) shows the concentration dependence of Young's modulus and tensile strength at $2.0 \times 2.0$ and $6.0 \times 6.0$. The results indicate that even for the specimens with draw ratio $2.0 \times 2.0$, Young's modulus and tensile strength are more pronounced with increasing concentration. This result supports the importance of the number of entanglements within the drawn film in Table III (a) to improve mechanical properties.

As listed in Table III(a), an apparent problem is that Young's modulus and tensile strength, even for the films with draw ratio $>7.0 \times 7.0(=49)$, are less than $4.5 \mathrm{GPa}$ and $0.2 \mathrm{GPa}$, respectively, while the Young's modulus and tensile strength corresponding to uniaxial films with a draw ratio of 50 times were $25-30 \mathrm{GPa}$ and $0.8-$ $1 \mathrm{GPa}$, respectively. ${ }^{29}$ Even so, the values of the Young's modulus and tensile strength of simultaneous biaxially stretched films are higher than the corresponding values
Table III. Young's modulus and tensile strength of simultaneous biaxially stretching films

(a) Young's modulus and tensile strength at the maximum draw ratio of the specimens prepared from the solutions with the indicated concentrations

\begin{tabular}{|c|c|c|c|}
\hline $\begin{array}{l}\text { Maximum } \\
\text { draw ratio }\end{array}$ & Concentration & $\begin{array}{l}\text { Young's } \\
\text { modulus }\end{array}$ & $\begin{array}{l}\text { Tensile } \\
\text { strength }\end{array}$ \\
\hline$\lambda_{\max }$ & $\mathrm{g} / 100 \mathrm{~mL}$ & $\mathrm{GPa}$ & $\mathrm{GPa}$ \\
\hline $7.3 \times 7.3$ & 0.7 & 2.5 & 0.12 \\
\hline $7.7 \times 7.7$ & 0.8 & 3.5 & 0.14 \\
\hline $8.7 \times 8.7$ & 0.9 & 4.3 & 0.16 \\
\hline $7.8 \times 7.8$ & 1.0 & 4.4 & 0.19 \\
\hline
\end{tabular}

(b) Young's modulus and tensile strength as a function of concentration of solutions to prepare gels

\begin{tabular}{|c|c|c|c|}
\hline Draw ratio & Concentration & Young's modulus & Tensile strength \\
\hline$\lambda$ & $\mathrm{g} / 100 \mathrm{~mL}$ & $\mathrm{GPa}$ & $\mathrm{GPa}$ \\
\hline \multirow{6}{*}{$2.0 \times 2.0$} & 0.4 & 1.8 & 0.05 \\
\hline & 0.6 & 1.9 & 0.07 \\
\hline & 0.7 & 2.0 & 0.08 \\
\hline & 0.8 & 2.1 & 0.09 \\
\hline & 0.9 & 2.2 & 0.10 \\
\hline & 1.0 & 2.3 & 0.11 \\
\hline \multirow{5}{*}{$6.0 \times 6.0$} & 0.6 & 1.9 & 0.09 \\
\hline & 0.7 & 2.2 & 0.10 \\
\hline & 0.8 & 3.2 & 0.12 \\
\hline & 0.9 & 3.6 & 0.13 \\
\hline & 1.0 & 3.9 & 0.14 \\
\hline
\end{tabular}

of $0.1 \mathrm{GPa}$ and $0.08 \mathrm{GPa}$ of the commercial blown films (crystallinity $52 \%$, melting point $138^{\circ} \mathrm{C}$ ). To check the temperature dependence of the mechanical property, the storage and loss moduli are estimated as a function of temperature.

Figure 2 shows the temperature dependence of storage and loss moduli for the film with a maximum draw ratio of $8.7 \times 8.7$ and for a commercial film. Because of mechanical anisotropy, the measurements were done in the machine and transverse directions. In a preliminary experiment, X-Ray diffraction patterns from through and end views revealed that the commercial film has the preferential orientation of the $a$-axes with respect to the machine direction but the crystallites has an uniaxial orientation around the machine direction. The storage modulus for both the films decreased with increasing temperature. The storage modulus of the biaxially stretched film is higher than that in the machine direction of commercial film in the given temperature range. The storage modulus of the biaxially stretching film at $23^{\circ} \mathrm{C}$ is $2.3 \mathrm{GPa}$, which is much lower than the modulus $(30 \mathrm{GPa})$ of the uniaxially stretched film with 50 -fold. For the loss modulus, the crystal dispersion (the $\alpha$ mechanism) for the gel film is observed around $70^{\circ} \mathrm{C}$, while the dispersion is indistinct for the commercial film because of the low crystallinity. The $\alpha$ mechanism is associated with grain boundary phenomena due to the rotation of crystal grains, as well as associated with the smearing-out effect of the crystal lattice potential due to onset of rotational oscillation of polymer chains within the crystal grain. ${ }^{31}$ The peak height becomes lower with decreasing concentration of solution during gel preparation, which is not shown in this paper. In contrast, a peak of the $\beta$ mechanism associated with molecular motion in the amorphous phase ${ }^{31}$ could be observed around 

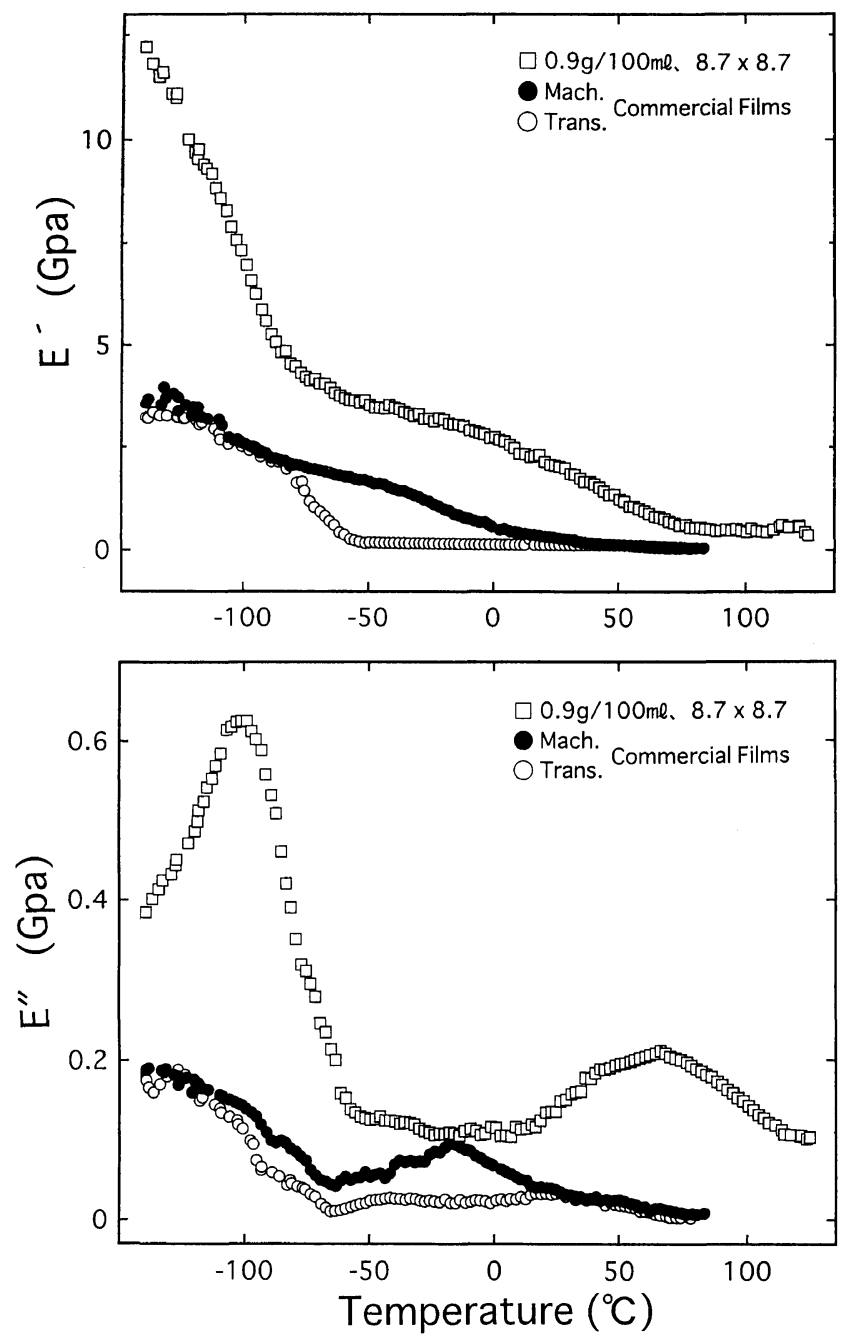

Figure 2. Temperature dependence of storage and loss moduli for the biaxial stretching films with draw ratio of $8.7 \times 8.7$ and commercial film.

$-20^{\circ} \mathrm{C}$ for the commercial film, but not observed for the gel film. The $\gamma$ dispersion peak appeared around $-100^{\circ} \mathrm{C}$ for the dry gel film and the commercial film, as has been reported as the local motions of side groups in the amorphous region and/or defects in the crystalline phase. The magnitude of the $\gamma$ mechanism is much higher than that of the $\alpha$ mechanism. Such a tendency is quite different from that of uniaxially stretched films. ${ }^{27}$ In accordance with previous results for uniaxially stretching, the magnitude of the $\alpha$ mechanism was highest among the three mechanisms and the magnitude became higher with increasing draw ratio indicating an increase in crystallinity. Anyway, Young's modulus of the biaxially stretching films is much lower than that of the uniaxially stretching films at the same draw ratio. This reason is discussed in terms of theoretical aspects.

\section{Morphology}

Figure 3 shows the reduced viscosity concentration relationship, in which (a) reveals the profile in the concentration range from 0 to $0.62 \mathrm{~g} / 100 \mathrm{~mL}$ and $(\mathrm{b})$ the detailed profile of the concentration lower than $0.2 \mathrm{~g} / 100$ $\mathrm{mL}$. The plot can be classified into four reduced viscosity regimes, very low concentration (lower than $0.1 \mathrm{~g} / 100$
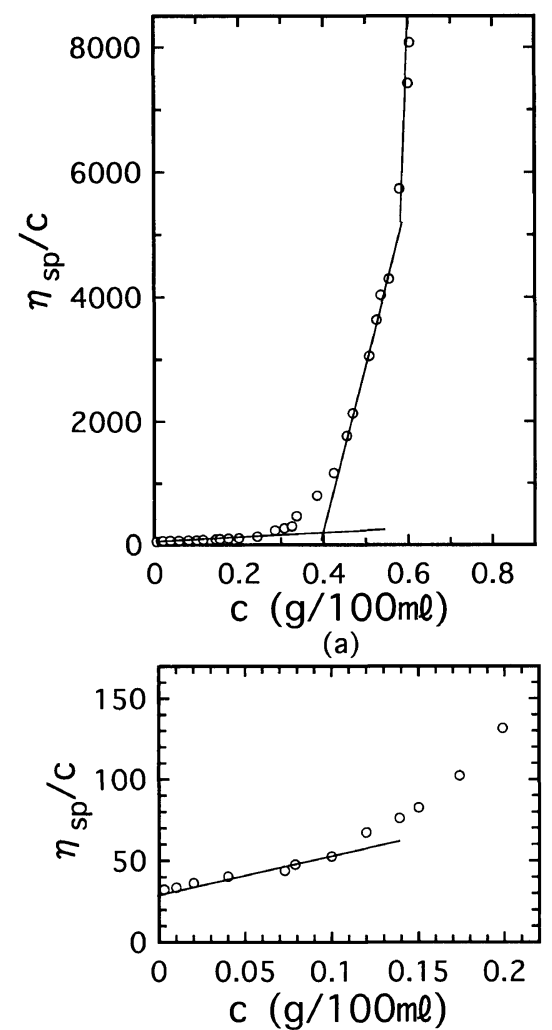

(b)

Figure 3. Reduced viscosity $v s$. concentration relationship.

$\mathrm{mL}$ ), low concentration (between 0.1 and $0.4 \mathrm{~g} / 100 \mathrm{~mL}$ ), high concentration (between 0.4 and $0.57 \mathrm{~g} / 100 \mathrm{~mL}$ ), and very high concentration (higher than $0.6 \mathrm{~g} / 100 \mathrm{~mL}$ ). Beyond $0.6 \mathrm{~g} / 100 \mathrm{~mL}$, the measurement was impossible, because of the extremely high viscosity. The optimum concentration to prepare the films assuring the greatest drawability under uniaxially stretching was $0.4-0.45 \mathrm{~g} /$ $100 \mathrm{~mL}$, while that under simultaneous biaxially stretching was $0.9 \mathrm{~g} / 100 \mathrm{~mL}$ corresponding to the very high viscosity regime. To study this reason, the morphology of the dry gels was estimated by SAXS, SEM, and WAXD.

Figure 4 shows SAXS and WAXD patterns for the original (un-drawn) dry gel films, when the incident beam was directed parallel to the film surface (end view). The WAXD patterns reveal the preferential orientation of the $c$-axes perpendicular to the film surface. The SAXS patterns show the meridional scattering having scattering maxima. The WAXD and SAXS patterns together indicate that the dried gel film is composed of crystal lamellae that are highly oriented with their large flat faces parallel to the film surface and within the lamellar crystals constituting the gel, the $c$-axes are oriented perpendicular to the large flat faces. Thus when the as-cast gel films are dried by slow evaporation of the solvent, the constituent lamellar crystals become oriented parallel to the film surfaces in a manner similar to mats of single crystals.

Figure 5 shows SAXS intensity distributions in the meridional direction for the films prepared by gelation/ crystallization from solutions with the indicated concentrations, when the incident beam was directed parallel 

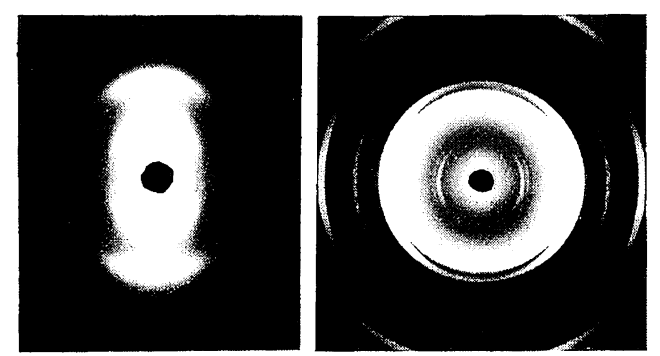

(a) $0.45 \mathrm{~g} / 100 \mathrm{ml}$
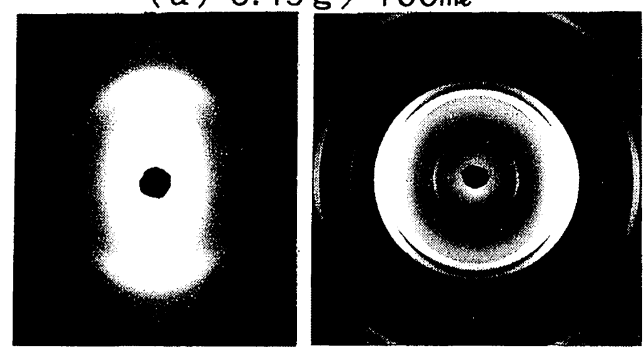

(b) $0.6 \mathrm{~g} / 100 \mathrm{ml}$
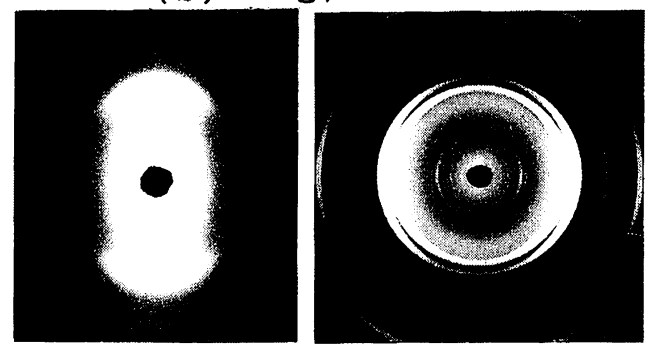

(c) $0.7 \mathrm{~g} / 100 \mathrm{ml}$
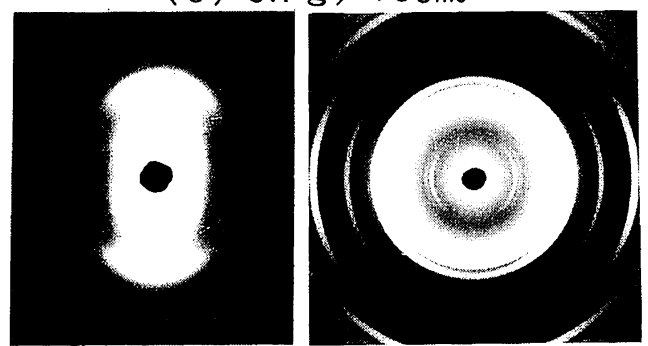

(d) $0.9 \mathrm{~g} / 100 \mathrm{ml}$

Figure 4. SAXS and WAXD patterns for the undrawn dry gel films.

to the film surface (end view). As discussed before, 0.45 and $0.9 \mathrm{~g} / 100 \mathrm{~mL}$ correspond to the optimum concentrations to assure the greatest significant drawability under uniaxially and simultaneous biaxially stretching, respectively. To obtain the intensity distribution, we have had a lot of efforts to make a sample holder in which a number of thin sheets $(>500)$ can be overlapped. It is of interest to note that the scattered maxima become distinct and move slightly close to the center of the scattering angle as the concentration decreases. Namely, the scattered intensity distributions of films prepared from solutions with 0.45 and $0.6 \mathrm{~g} / 100 \mathrm{~mL}$ show up to the fourth order maxima, while those, with $0.7,0.8,0.9$, and $1.0 \mathrm{~g} / 100 \mathrm{~mL}$ show up to the third order maxima.

At $0.45 \mathrm{~g} / 100 \mathrm{~mL}$, most of chain molecules in solution are random coils having coupling entanglements which will be predominantly intra-molecular in nature but have a suitable level of the entanglements that act as inter-lamellar cross-links and effectively transmit the drawing force under uniaxially stretching. On the other

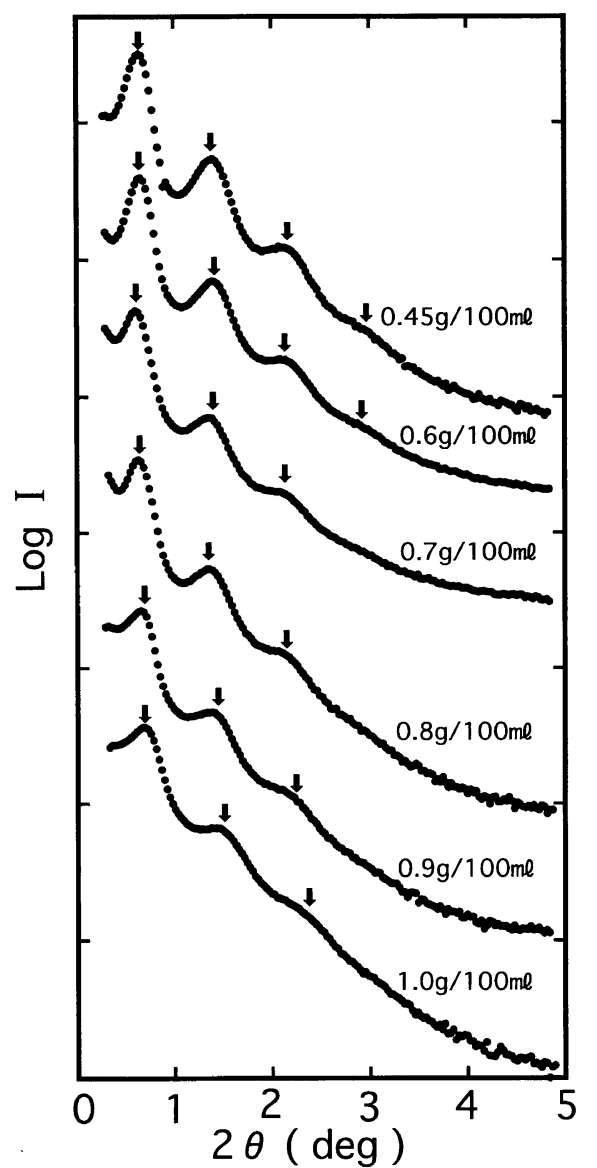

Figure 5. SAXS intensity distribution in the meridional direction for the films prepared from solutions with the indicated concentration.

hand, solutions corresponding to the regime of high concentration are thought to consist of interpenetrating random coils that form a large number of coupling entanglements that are both intra- and inter-molecular. Accordingly, the molecular chains cannot form large lamellae because of a large number of entanglements; the resulting small lamellar crystals are inter-connected by the chain molecules. The profile of the SAXS intensity distribution satisfies this concept; the long period becomes shorter and each height is lower with increasing concentration. Such a condition is found to be suitable for simultaneous biaxially stretching, which is different from the results obtained for uniaxially stretching.

In spite of the change in profile with concentration, the dry gel films composed of lamellar crystals stacked on top of one another to form a periodic structure perpendicular to the plane of the film. Such system is not so difficult in formulating an equation of scattered intensity distribution. In previous works on SAXS intensity distribution, the theoretical calculation was carried out for two cases; one is that the scattering is caused by the excess electron density ${ }^{32,33}$ and the other is that the scattering is caused by positive and negative deviation from the average density of the sample. ${ }^{34,35}$ The latter case is a somewhat complicated but realistic system. Considering the latter case, the equation of scattered intensity contains a number of parameters and it was very difficult to estimate an exact value for all parameters by 
computer. In order to avoid such trouble, the orientational fluctuation of crystal lamellae and fluctuation of lamellar size were neglected and the analysis was limited for two phase structures composed of completely oriented lamellar micro-domains.

Based on this assumption, attention was focused on a quantitative estimation of the boundary (transition) regions $t$ corresponding to folded loops on the basis of SAXS intensity distributions at larger scattering angles than those in Figure 5. On subtracting the background scattering arising from the amorphous order and thermal density fluctuation from the total scattered intensity, the background scattering was approximated as a straight line. Through trial and error, however, it was found that the manner of subtracting the background scattering did not much affect the final value of the density fluctuation at the interface.

The system to be considered here is the diagram that has one-dimensional electron density fluctuation along a direction normal to the lamellar interfaces and the density variation is periodic. The variation deviates from the variation of an ideal two-phase system in which the density variation occurs discontinuously from electron density of crystal lamellae and voids.

If the electron density variation is given by a Gaussian function, the scattered intensity at larger angle tail $I(s)$, corrected for background scattering, is given by ${ }^{36,37}$

where

$$
I(s)=(\text { const }) s^{-2} \exp \left(-4 \pi^{2} \sigma^{2} s^{2}\right)
$$

$$
s=\frac{2 \sin \theta}{\lambda^{\prime}}
$$

where $\lambda^{\prime}$ is the wavelength of the X-Ray and $2 \theta$ is the scattering angle. $\sigma$ is the parameter denoting standard deviation characterizing the diffuseness of the boundary and it is given the interfacial thickness $t$, as follows:

$$
\sigma=(2 \pi)^{-1 / 2} t
$$

The value $t$ can be evaluated from the slope in the plot of $\operatorname{In}\left(s^{2} I(\mathrm{~s})\right)$ vs. $s^{2}$. Figure 6 shows the results for three kinds of dry gel films.

Table IV summarizes the periodic distance $L$ evaluated from Figure 5 and the interfacial thickness $t$ evaluated from Figure 6. The concentration gives significant change in $L$ but not in $t . L$ decreases with increasing the concentration but $t$ is almost constant. This is out of our expectation. Namely, since there exist a number of entanglements in a $0.9 \mathrm{~g} / 100 \mathrm{~mL}$ solution, it was expected that the resultant films provide larger interfacial region in comparison with the films prepared from solutions with low concentrations $(0.4-0.45 \mathrm{~g} / 100 \mathrm{~mL})$. This discrepancy still remains as an unresolved problem.

Figure 7 shows the $67.5 \mathrm{MHz}$ CPMAS and PSTMAS ${ }^{13} \mathrm{C}$ NMR spectra of the biaxial films with a draw ratio of $8.7 \times 8.7$ and of the uniaxial films with a draw ratio of 70. CPMAS spectra emphasize the contribution of crystalline phase, while PSTMAS spectra, the contribution of amorphous chain motion by NOE (nuclear Overhauser effect). The CPMAS spectrum (a) of biaxially stretching film shows the two peaks. The peaks ap-

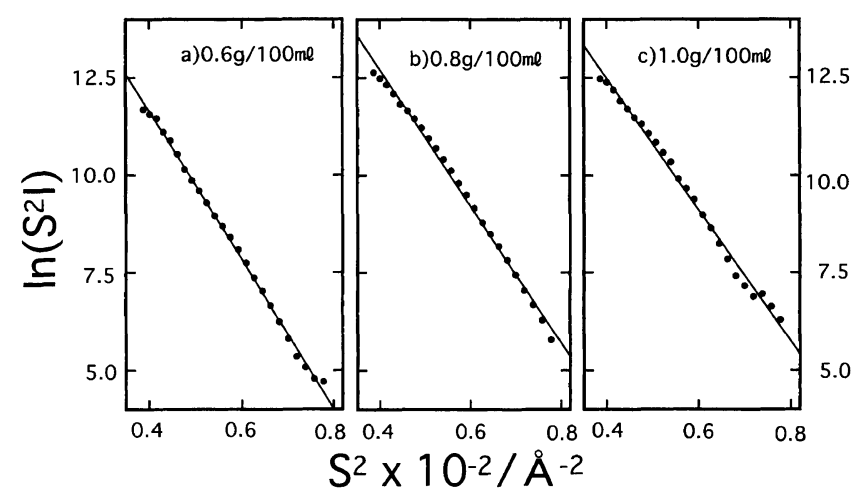

Figure 6. Full analysis of the interfacial thickness, $\ln \left(s^{2} I(s)\right)$ $v s . s^{2}$ of dry gel films prepared from solutions with the indicated concentrations.

Table IV. Periodic distance $L$ and interfacial thickness $t$ of

\begin{tabular}{|c|c|c|}
\hline Concentration & Periodic distance $L$ & nterfacial thickness $t$ \\
\hline $\mathrm{g} / 100 \mathrm{~mL}$ & $\AA$ & $\AA$ \\
\hline 0.45 & 112 & 15.0 \\
\hline 0.60 & 106 & 15.8 \\
\hline 0.70 & 103 & 15.8 \\
\hline 0.80 & 103 & 15.6 \\
\hline 0.90 & 103 & 15.6 \\
\hline 1.0 & 100 & 15.5 \\
\hline
\end{tabular}
undrawn dry gels prepared from solutions with the indicated concentrations

peared at 32.41 and $30.76 \mathrm{ppm}$, respectively. The former peak corresponds to the orthorhombic crystal form component and the latter, the amorphous one. The PSTMAS spectrum (b) shows a shoulder at $32.51 \mathrm{ppm}$ associated with the contribution of the orthorhombic crystal form and a clear peak at $30.57 \mathrm{ppm}$ associated from the amorphous component. For the uniaxially stretched film, the CPMAS spectrum (c) shows two peaks. One is at 32.90 ppm associated with the orthorhombic crystal form component and the other, at $33.51 \mathrm{ppm}$ associated from monoclinic crystal form component. No peak from the amorphous component can be observed. The PSTMAS spectrum (d) shows a large peak at $32.99 \mathrm{ppm}$ associated with the orthorhombic crystal form component, while it shows a shoulder at $31.02 \mathrm{ppm}$ associated with the amorphous component, indicating that the molecular mobility within the uniaxially stretched film is much less than that within the biaxially stretched film because of higher crystallinity. These four NMR spectra indicate the difference of morphology between biaxially and uniaxially stretched films clearly. The biaxially stretched films have low crystallinity and high molecular chain mobility, while uniaxially stretched films have high crystallinity and poor molecular mobility. These results support the low crystallinity and low melting point of simultaneous biaxially stretched films shown in Tables II and III.

To look for the origin of the poor mechanical properties in a macroscopic sense, the change in the appearance of the specimens with low and high draw ratios under SEM is shown in Figure 8. The fibrillar texture of the film with $2.0 \times 2.0$ shows an indistinct uneven surface. At the maximum draw ratio of $8.7 \times 8.7$, the clear fi- 

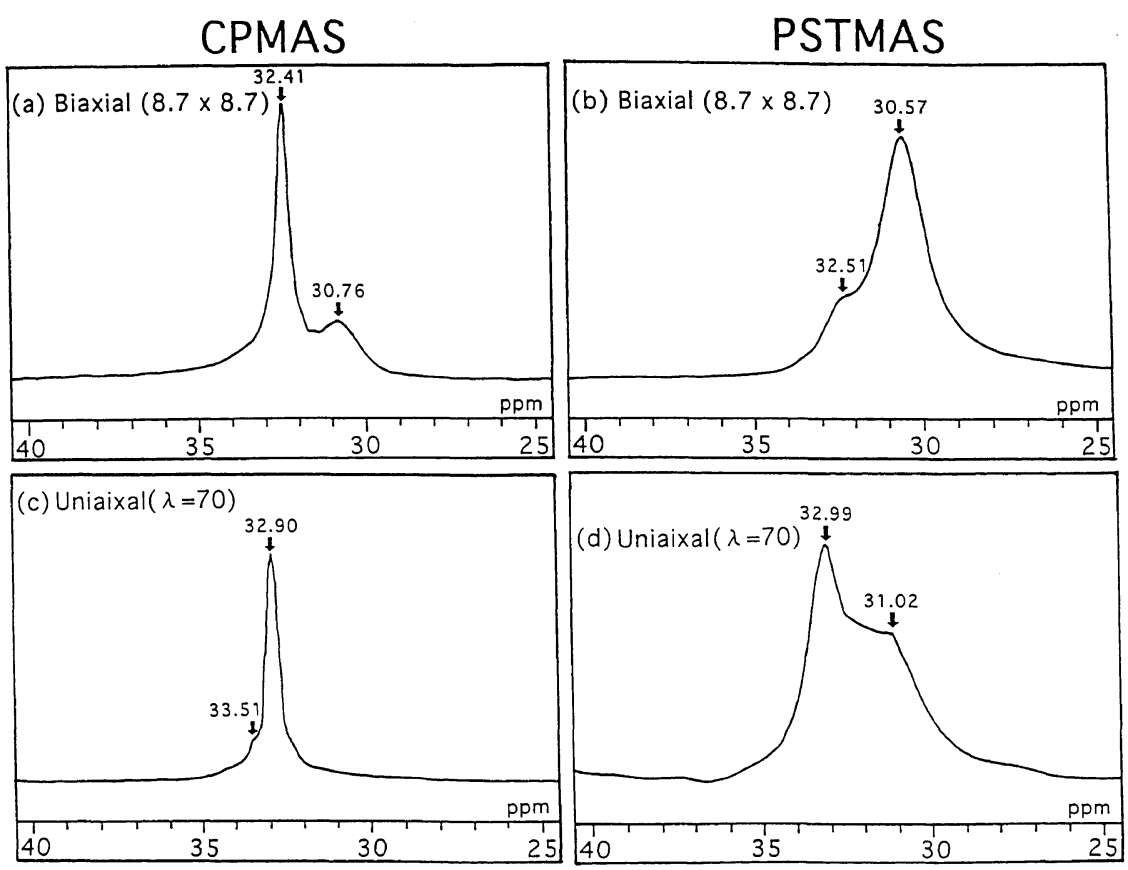

Figure 7. CPMAS and PSTMAS spectra of the biaxial stretching film with a draw ratio of $8.7 \times 8.7$ and the uniaxial stretching film with a draw ratio of 70 .
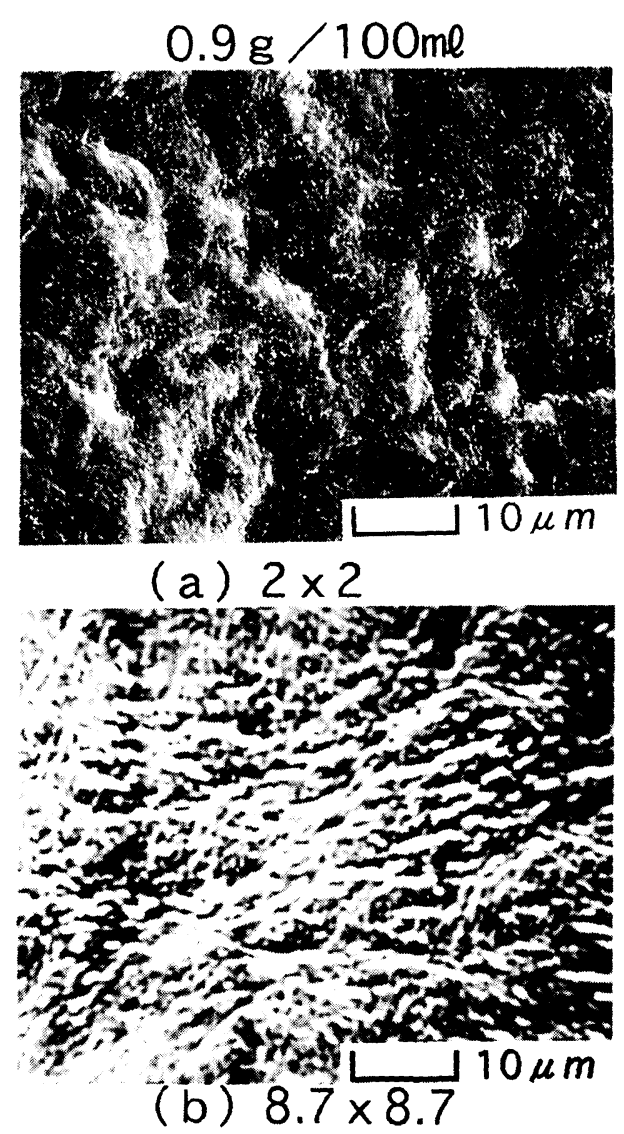

Figure 8. Scanning electron micrographs of the biaxial stretching films. (a) draw ratios of $2.0 \times 2.0$ and (b) draw ratio of $8.7 \times 8.7$.

brils could be observed but they were isolated without forming the network structure that has been observed for uniaxially stretched films. ${ }^{29,38}$ This indicates that the existence of a number of entanglements is important to avoid the cutting of fibrils as much as possible under the elongation process. Actually, the films prepared from solution with concentrations lower than $0.9 \mathrm{~g} / 100 \mathrm{~mL}$ showed more drastic cutting of fibrils under the biaxial stretching.

\section{Orientation of Crystallites within Biaxially Stretched Films}

Figure 9 shows WAXD patterns (end view) of drawn films. The patterns at the same draw ratio are almost the same, independent of concentration of solution. The (110) and (200) planes with an orientation perpendicular to film surface tend to orient parallel to the film surface. At $\lambda=2$, the (200) plane is oriented at $45^{\circ}$ with respect to the film thickness direction. With increasing $\lambda$, the (200) plane orients parallel to the film surface. In accordance with the orientational modes of the (200) plane, the (110) plane takes the two modes, which is similar to the orientational mode of 10 times biaxially drawn films reported by Gerrits and Young. ${ }^{18}$ They analyzed the crystal orientation in terms of crystal plasticity associated with both slip and twinning processes and hexagonal and martensitic phase transformations. Unlike their analysis, this paper deals with the deformation mechanism of biaxially drawn films in terms of orientation distribution function of crystallites and amorphous chain segments. In doing so, the orientation distribution function of crystallites must be obtained using the orientation distribution function of the reciprocal vectors of the crystal planes. For this purpose, we set following geometrical arrangements.

Figure $10 \mathrm{a}$ shows Cartesian coordinate $0-U_{1} U_{2} U_{3}$ fixed within a structural unit, with respect to another Cartesian coordinate $0-X_{1} X_{2} X_{3}$ fixed in a bulk specimen. The $U_{3}$ axis may be taken along the $c$-axis. Because of the simultaneous biaxially stretched film, the $c$-axes have a random orientation around the $X_{3}$ axis (the film 

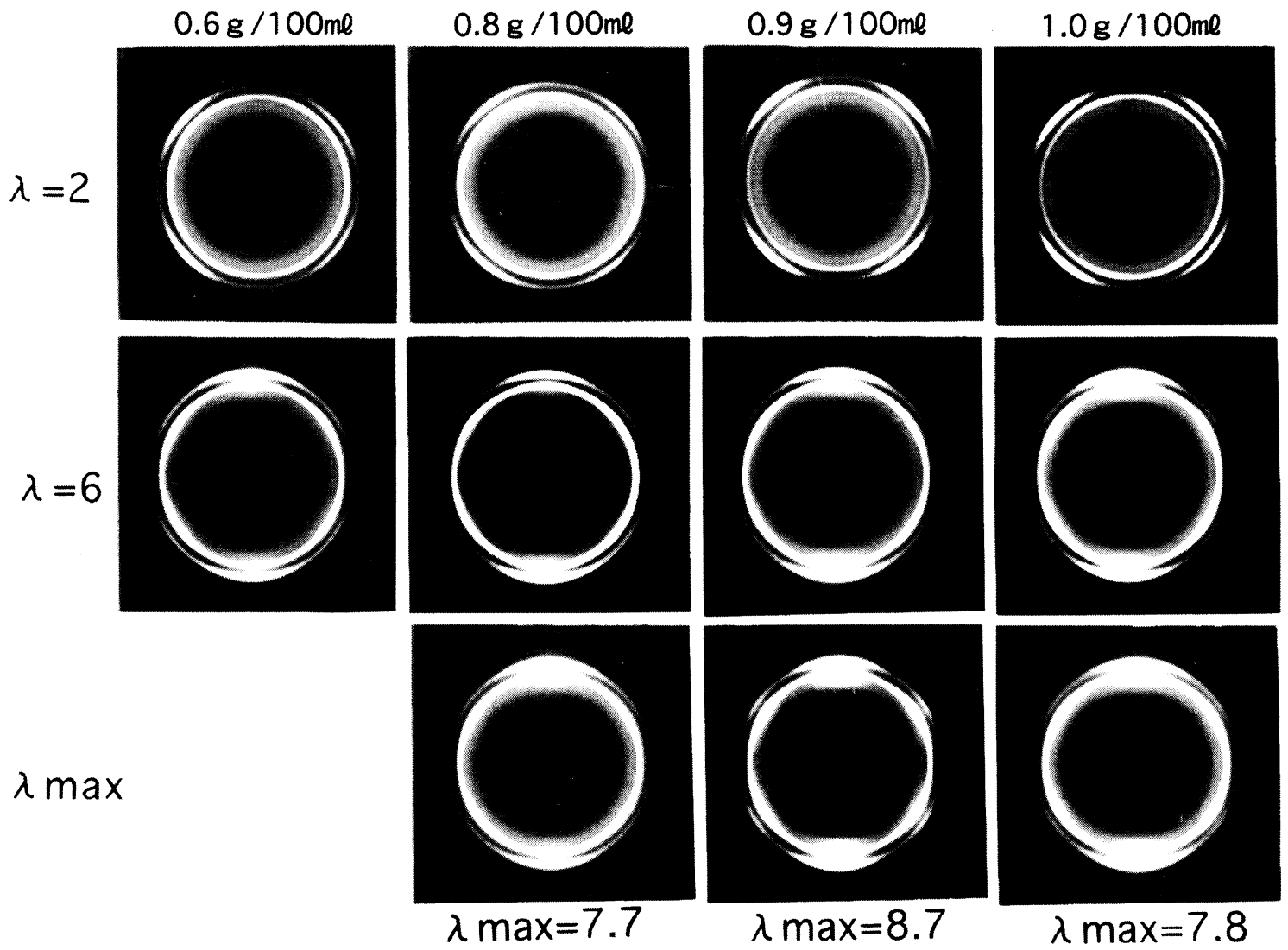

Figure 9. WAXD patterns (end view) of various drawn films.

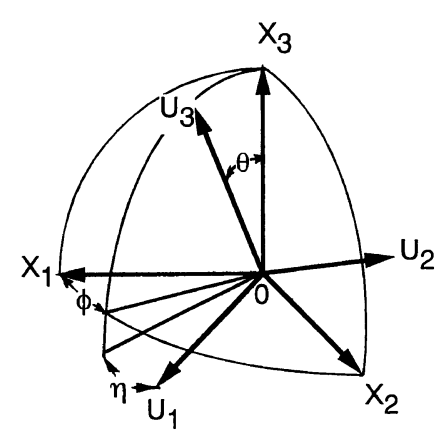

(a)

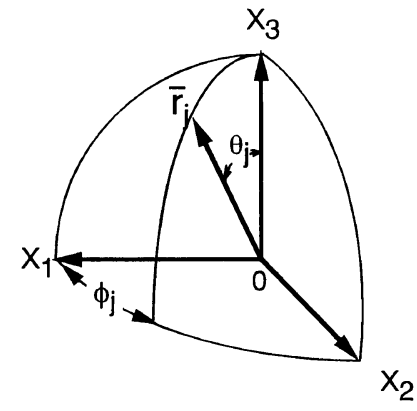

(b)

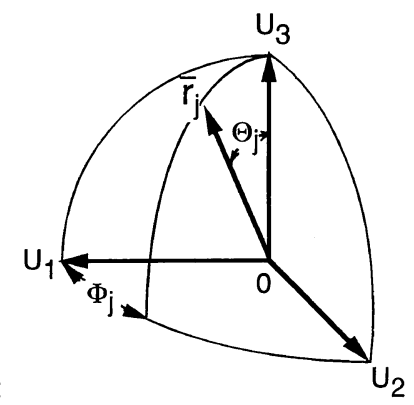

(c)

Figure 10. Cartesian coordinate illustrating the geometrical relation (a) Euler angles $\theta$ and $\eta$ which specify the orientation of coordinate $0-U_{1} U_{2} U_{3}$ of structural unit with respect to coordinate $0-X_{1} X_{2} X_{3}$ of specimen. (b) Angles $\theta_{j}$ and $\phi_{j}$ which specify the orientation of the given $j-$ th axis of the structural unit with respect to the coordinate $0-X_{1} X_{2} X_{3}$. (c) Angles $\Theta_{j}$ and $\Phi_{j}$ which specify the orientation of the $j$-th axis of the structural unit with respect to the coordinate $0-U_{1} U_{2} U_{3}$.

thickness direction) in the present system. The orientation of the structural unit within the space of the film specimen may be specified by using three Euler angles, $\phi, \theta$, and $\eta$. The angles $\theta$ and $\phi$, which define the orientation of the $U_{3}$ axis of the unit within the space, are polar and azimuthal angles, respectively, and $\eta$ specifies the rotation of the unit around its own $U_{3}$ axis. Coordinates (b) and (c) show a given $j$-th axis $r_{j}$ within the unit, specified by the polar angle $\theta_{j}$ and the azimuthal angle $\phi_{j}$ with respect to the Cartesian coordinate $0-X_{1} X_{2} X_{3}$ and specified by polar angle $\Theta_{j}$ and the azimuthal angle $\Phi_{j}$ with respect to the $0-U_{1} U_{2} U_{3}$ of the unit.

For an uniaxial system, the orientation distribution function $\omega(\theta, \eta)$ of crystallites may be calculated from the orientation distribution function of the reciprocal lattice vector of the $j$-th plane, $2 \pi q\left(\cos \theta_{j}\right)$, by using a method proposed by Roe and Krigbaum ${ }^{22-24}$

$$
\begin{aligned}
& F_{l 0}^{j}=\left\langle P_{l}\left(\cos \theta_{j}\right)\right\rangle=\int_{0}^{2 \pi} \int_{0}^{\pi} q_{j}\left(\cos \theta_{j}\right) P_{l}\left(\cos \theta_{j}\right) \sin \theta_{j} \mathrm{~d} \theta_{j} \mathrm{~d} \phi_{j} \\
& F_{l 0}^{j}=F_{l 00} P_{l}\left(\cos \Theta_{j}\right)+2 \sum_{n=2}^{l} \frac{(l-n) !}{(l+n) !} F_{l 0 n} P_{l}^{n}\left(\cos \Theta_{j}\right) \cos n \Phi_{j}
\end{aligned}
$$




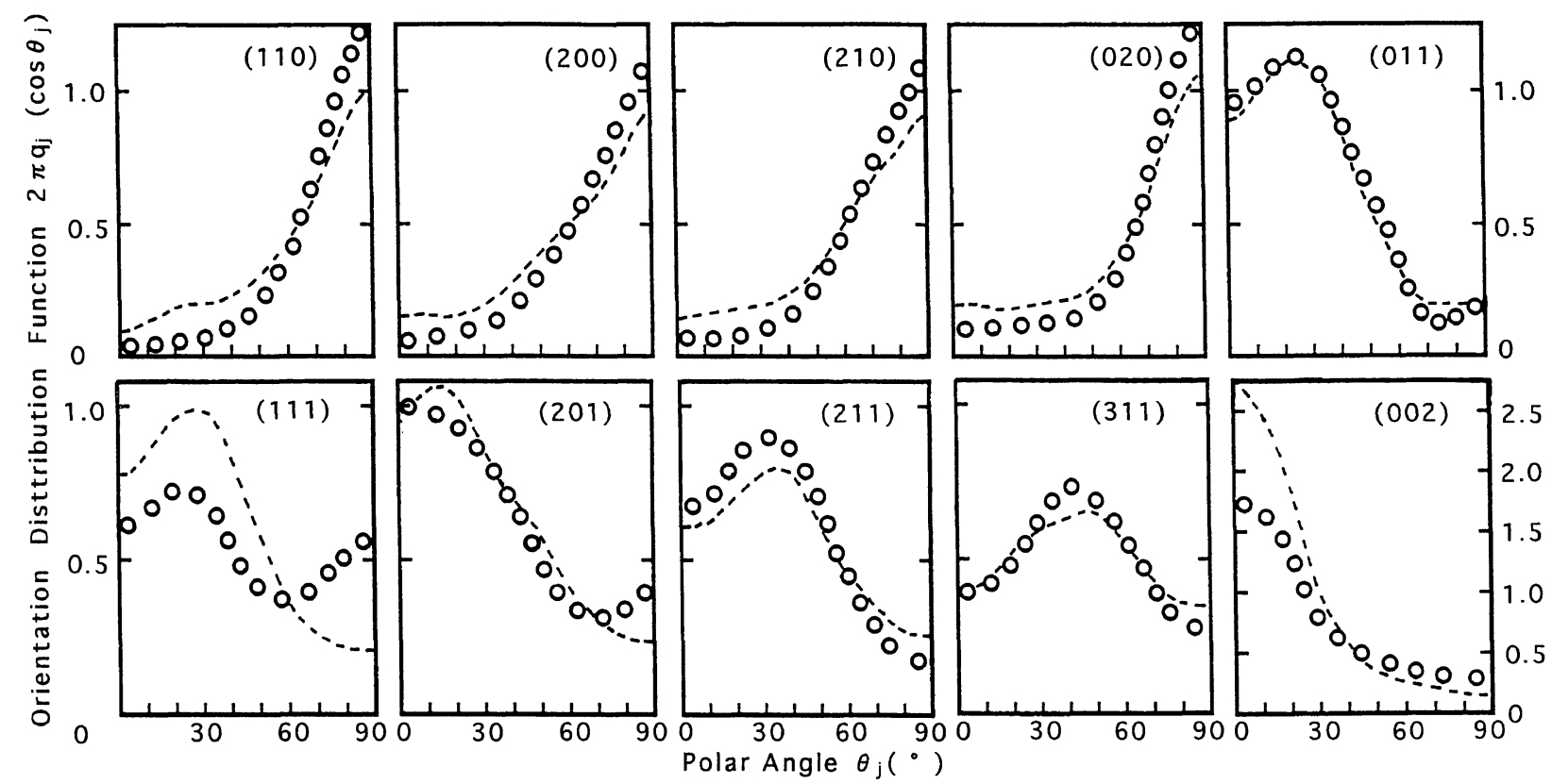

Figure 11. Orientation distribution functions $2 \pi q_{j}\left(\cos \theta_{j}\right)$ of the reciprocal lattice vectors of the indicated crystal planes of an undrawn polyethylene film. Circles: values of $2 \pi q_{j}\left(\cos \theta_{j}\right)$ obtained from experimental measurements. Dashed curves: calculated with the 21-term series.

$$
\begin{aligned}
4 \pi^{2} \omega(\cos \theta, \eta)=\frac{1}{2}+\sum_{l=2}^{\infty} & \frac{(2 l+1)}{2}\left\{F_{l 00} P_{l}(\cos \theta)\right. \\
& \left.+2 \sum_{n=2}^{l} \frac{(l-n) !}{(l+n) !} F_{l 0 n} \cos n \eta\right\}
\end{aligned}
$$

Here $l$ and $n$ are even integers. $P_{l}^{n}(X)$ and $P_{l}(X)$ are the associated Legendre polynomials and Legendre polynomials, respectively. $F_{l 0}^{j}$ and $F_{l 0 n}$ are the coefficients. $F_{l 0}^{j}$ is the $l$-th order orientation factor of the $j$-th crystal plane estimated by X-Ray diffraction, while the generalized orientation factor $F_{l 0 n}$ can be determined by solving the linear equations represented by eq 7 , since there exist more equations than the number of unknowns, as was pointed out by Roe and Krigbaum..$^{22-24}$

The values of weighing factor $\rho_{j}$, required in the leastsquares calculation, were assigned somewhat subjectively on the assumption that the X-Ray diffraction intensity is dependent upon the structure factor of each crystal plane. Hence, in this calculation, the weighing factors $\rho_{j}$, as a first approximation, were assumed to be almost proportional to the structure factor and were subsequently modified to obtain the best fit between experimental and calculated results through numerical calculations by computer. The calculation was continued until the best fit was achieved within the capability of the simplex method. ${ }^{39}$ Using the final values of parameters, a mean-square error between the calculated $F_{l 0}^{j}$ and recalculated $F_{l 0}^{j}$ was obtained using:

$$
R=\frac{\left.\sum_{j} \sum_{l} \rho_{j}\left[\left(F_{l 0}^{j}\right)_{\text {cal }}\right]-\left(F_{l 0}^{j}\right)_{\text {recal }}\right]^{2}}{\sum_{j} \sum_{l}\left[\left(F_{l 0}^{j}\right)_{\text {cal }}\right]^{2}}
$$

Figures 11-13 compare the observed orientation dis- tribution functions $2 \pi q_{j}\left(\cos \theta_{j}\right)$ with those recalculated for the respective crystal planes for the films with $\lambda=$ $1.0 \times 1.0$ (the undrawn film), $2.0 \times 2.0$, and $8.7 \times 8.7$, respectively, which were prepared from a $0.9 \mathrm{~g} / 100 \mathrm{~mL}$ solution. We calculated $F_{l 0}^{j}$ from eq7 to minimize the value of $R$ in eq 9 . After that, we recalculated $F_{l 0}^{j}$, in turn, from the values of $F_{l o n}$, and further calculated $2 \pi q_{j}$ $\left(\cos \theta_{j}\right)$ from the recalculated $F_{l 0}^{j}$ value using the following equation:

$$
2 \pi q_{j}\left(\cos \theta_{j}\right)=\frac{1}{2}+\sum_{l=2}^{\infty} \frac{2 l+1}{2} F_{l 0}^{j} P_{l}\left(\cos \theta_{j}\right)
$$

The values of $R$ in eq 9 were $7.5 \%$ at $\lambda=1.0,8.3 \%$ at $\lambda$ $=2.0 \times 2.0$, and $8.9 \%$ at $\lambda=8.7 \times 8.7$.

Returning to Figures $11-13$, it is evident that fairly good agreement between the observed and calculated distribution functions of the reciprocal lattice vectors of the indicated crystal planes was obtained, even for less accurately measured crystal planes with lower weighing factors. In Figure 11, $2 \pi q_{j}\left(\cos \theta_{j}\right)$ for the $(\mathrm{h} \mathrm{k} 0)$ planes at $\lambda=1$ has a maximum at $\theta_{j}=90^{\circ}$ with the same magnitude and that for the (002) plane has a peak at $\theta_{j}=0^{\circ}$. This indicates that the $c$-axes orient predominantly in the film thickness direction and crystallites take random rotation around their own $c$-axes. In Figure 12, $2 \pi q_{j}\left(\cos \theta_{j}\right)$ concerning the $(200)$ and $(002)$ planes at $\lambda=$ $2.0 \times 2.0$ have a sharp peak at $45^{\circ}$, while $2 \pi q_{j}\left(\cos \theta_{j}\right) \operatorname{con}$ cerning the $(020)$ plane has a maximum at $90^{\circ}$ indicating that the $b$-axes are oriented predominantly in the stretching direction. This means that such orientation of the $c$-axes and $a$-axes is attributed to the rotation of crystallites around their own $b$-axes. In Figure 13, $2 \pi q_{j}\left(\cos \theta_{j}\right)$ concerning the (200) plane for the film at $\lambda=$ $8.7 \times 8.7$ has a maximum peak at $\theta_{j}=0^{\circ}$ indicating the preferential orientation of the $a$-axes perpendicular to 


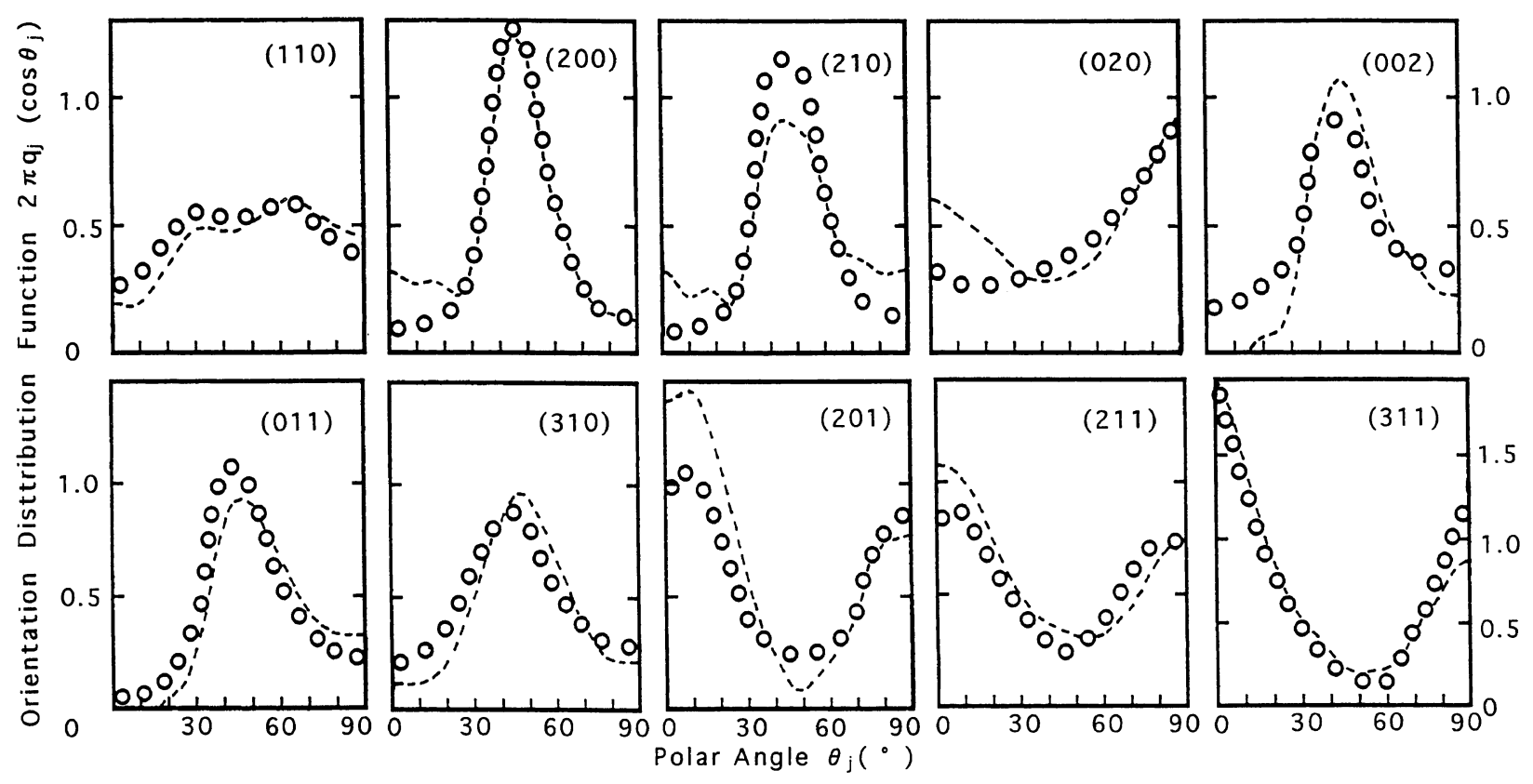

Figure 12. Orientation distribution functions $2 \pi q_{j}\left(\cos \theta_{j}\right)$ of the reciprocal lattice vectors of the indicated crystal planes of a drawn polyethylene film with draw ratio of $2.0 \times 2.0$. Circles: values of $2 \pi q_{j}\left(\cos \theta_{j}\right)$ obtained from experimental measurements. Dashed curves: calculated with the 21-term series.

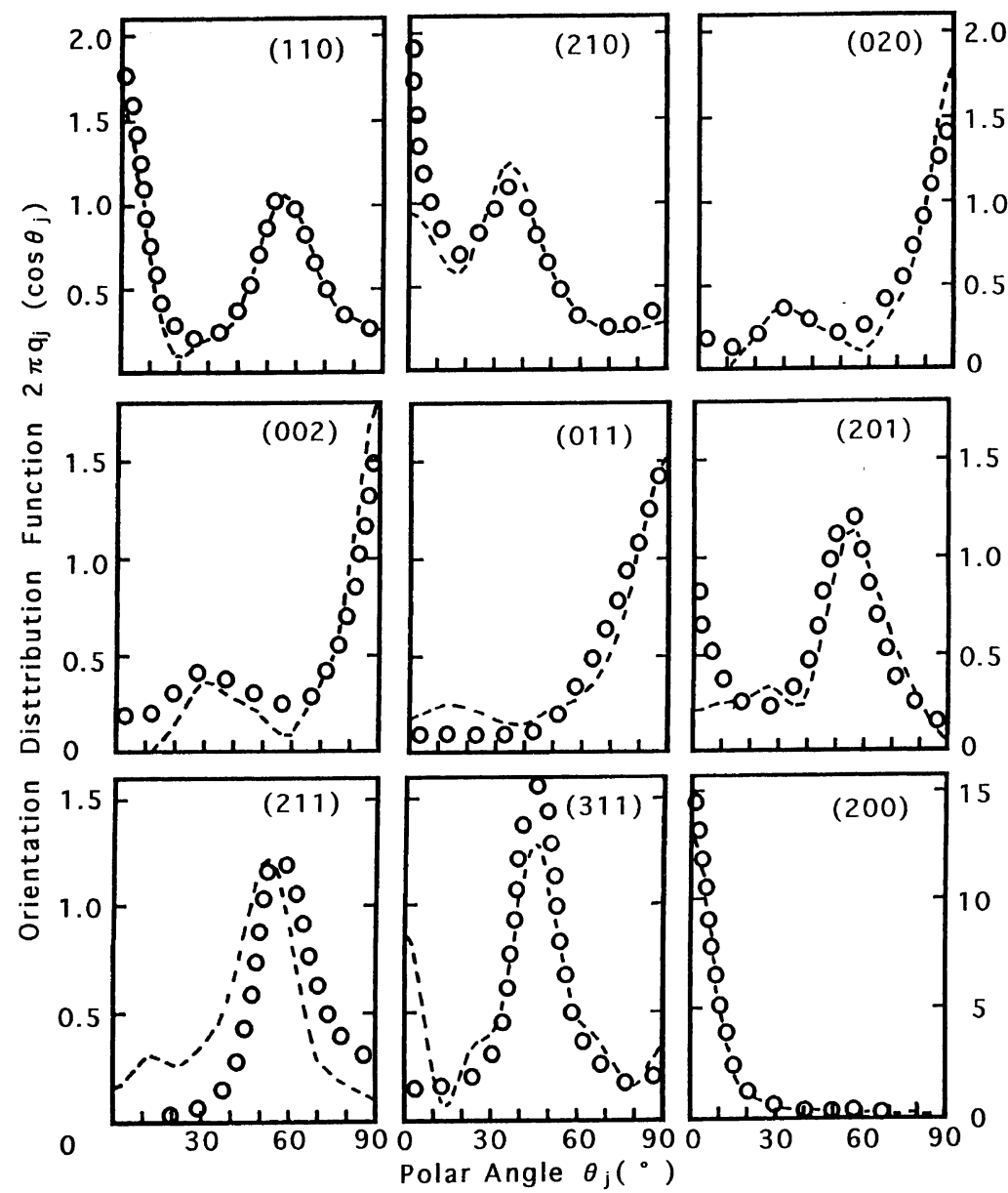

Figure 13. Orientation distribution functions $2 \pi q_{j}\left(\cos \theta_{j}\right)$ of the reciprocal lattice vectors of the indicated crystal planes of a drawn polyethylene film with draw ratio of $8.7 \times 8.7$. Circles: values of $2 \pi q_{j}\left(\cos \theta_{j}\right)$ obtained from experimental measurements. Dashed curves: calculated with the 21-term series. 
the film surface. In contrast, the function of the (002) plane has two peaks at $\theta_{j}=90^{\circ}$ and $30^{\circ}$ indicating the two orientational modes. The large peak at $90^{\circ}$ denotes that the $c$-axes orient mainly in the stretching direction, while the small peak at $30^{\circ}$ denotes the orientation of a few crystallites whose $c$-axes orient at $60^{\circ}$ predominantly with respect to the stretching direction. The orientation of the $b$-axes takes a large peak at $\theta_{j}=90^{\circ}$ and a small peak at $\theta_{j}=30^{\circ}$ indicating orientational mode similar to the $c$-axes. Such a complicated behavior indicates that the orientation of crystallites has at least two modes. The main mode is the preferential orientation of the $c$-axis by the rotation around the $b$-axis but the other minor mode is not precisely known. Accordingly, the orientation distribution functions of crystallites were represented to give a quantitative analysis for the complicated behavior.

Figure 14 (a), (b), and (c) show the results for the films with $\lambda=1.0 \times 1.0,2.0 \times 2.0$, and $8.7 \times 8.7$, respectively. In map (a), the magnitude is the highest at $\theta=0^{\circ}$ and no appreciable $\eta$-dependence is observed, indicating that the crystallites rotate randomly around their own $c$ axes. Hence it is evident that the $c$-axes are oriented parallel to the film thickness direction like single crystal mats. In map (b), the density is the highest at $\theta=45^{\circ}$ and $\eta=0$ indicating that when the specimen is elongated up to $\lambda=2.0 \times 2.0$, the $c$-axes orient at $\theta=45^{\circ}$ with respect to the stretching direction by rotating around their $b$-axes, leading to the occurrence of shear stress. Map (c) shows a complicated profile with several density maxima. Among them, the density at $\theta=90^{\circ}$ and $\eta=0^{\circ}$ are highest. Such orientation is due to the fact that the $c$-axes orient predominantly parallel to the film surface by the rotation of crystallites around the $b$ axes with the further elongation from $\lambda=2.0 \times 2.0$ to 8.7 $\times 8.7$. The other density maxima, for example, at $\theta=90^{\circ}$ and $\eta=50^{\circ}$ and $\theta=55^{\circ}$ and $\eta=90^{\circ}$ are thought to be due to the contribution from the small peak appeared at $\theta_{j}=30^{\circ}$ of $2 \pi q_{j}\left(\cos \theta_{j}\right)$ for the (020) and (002) planes in Figure 13. However, such a profile is too complicated to obtain more detailed information and further analysis must be done in future studies.

In order to characterize the orientation behavior of crystallites within the dry gel films, the second order orientation factor of the amorphous chain segments was obtained from the birefringence as estimated by subtraction of the crystalline contribution from the total birefringence, assuming simple additivity as indicated in the following equation: ${ }^{40}$

$$
\Delta_{\text {total }}=X_{\mathrm{c}} \Delta_{\mathrm{c}}+\left(1-X_{\mathrm{c}}\right) \Delta_{\mathrm{a}}+\Delta_{\mathrm{f}}
$$

where $\Delta_{\text {total }}$ is the total birefringence of the bulk specimen, $\Delta_{c}$ is the crystalline birefringence, is the noncrystalline birefringence, $X_{\mathrm{c}}$ is the volume fraction of crystalline phase, and $\Delta_{\mathrm{f}}$ is the form birefringence. $\Delta_{\mathrm{c}}$ and $\Delta_{\mathrm{a}}$ are given by

$$
\begin{aligned}
\Delta_{\mathrm{c}} & =n_{\mathrm{a}} F_{200}^{[200]}+n_{\mathrm{b}} F_{200}^{[020]}+n_{\mathrm{c}} F_{200}^{[002]} \\
& =\left(n_{\mathrm{c}}-n_{\mathrm{a}}\right) F_{200}^{[002]}+\left(n_{\mathrm{b}}-n_{\mathrm{a}}\right) F_{200}^{[020]}
\end{aligned}
$$

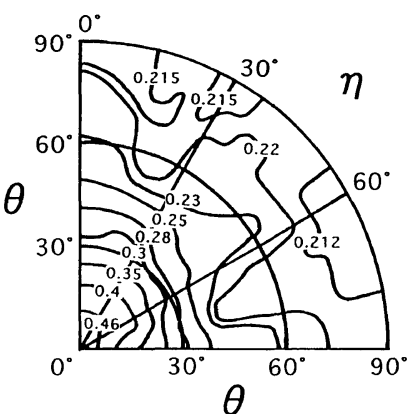

(a) Undrawn

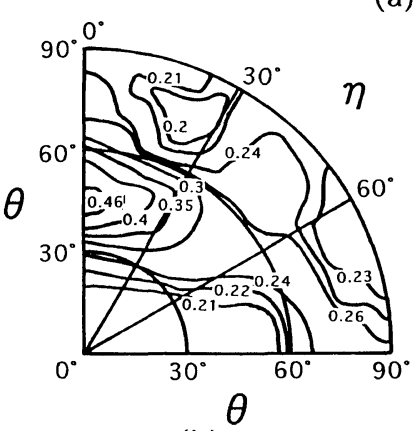

(b) $2 \times 2$

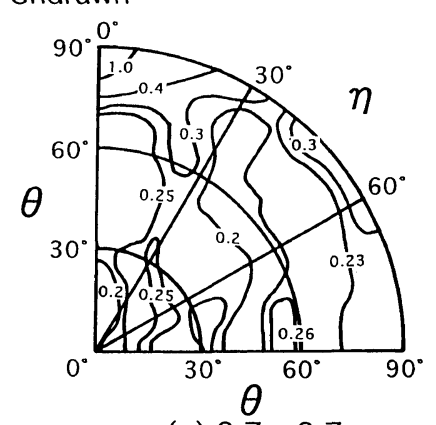

(c) $8.7 \times 8.7$
Figure 14. Orientation distribution functions of crystallites $4 \pi^{2} \omega(\theta, \eta)$ calculated by eqs $6-8$. (a) undrawn film (b) drawn film with $2.0 \times 2.0$ (c) drawn film with $8.7 \times 8.7$.

$$
\Delta_{\mathrm{a}}=\left(n_{\|}-n_{\perp}\right) F_{200}^{a m}
$$

where $n_{\mathrm{a}}, \mathrm{n}_{\mathrm{b}}$, and $\mathrm{n}_{\mathrm{c}}$ are the refractive indices along the $a, b$, and $c$-axes, which are given as $1.514,1.519$, and 1.575 , respectively. ${ }^{41} n_{\|}$and $n_{\perp}$ are the refractive indices parallel and perpendicular to an amorphous chain segment. The birefringence of $\left(n_{\|}-n_{\perp}\right)$ of the amorphous chain segment is $52 \times 10^{-3}$ and $F_{200}^{a m}$ is the second order orientation factor. The orientation factors, $F_{20}^{j}$ and $F_{200}^{a m}$, characterize the molecular orientation distribution with variation between $-1 / 2$ and 1 . In a simultaneous biaxially stretched film, they are 0 for random orientation, while for complete orientation parallel and perpendicular to the film normal direction, they are unity and $-1 / 2$, respectively. In other words, when the molecular chains are perfectly oriented along the stretching direction, they are $-1 / 2$.

By neglecting $\Delta_{\mathrm{f}}$, the orientation factors of the $c$-axes and the amorphous chain segments may be obtained through eq $11-13$. For the film with $2.0 \times 2.0, F_{200}$ for crystallites and $F_{200}^{a m}$ for amorphous chain segments are 0.0341 and -0.0779 , respectively, while for the film with $8.7 \times 8.7, F_{200}$ and $F_{200}^{a m}$ are -0.173 and -0.341 , respectively. The preferential orientational degree of the amorphous chain segments is higher than that of the $c$ axes under simultaneous biaxially stretching. This indicates that the preferential orientation of the $c$-axes arisen by the rotation of crystallites around their $b$-axes to the stretching direction is due to straining of tie molecules. This process accompanies the crystal transformation from a folded to a fibrous type at initial draw ratio $<$ $2.0 \times 2.0$, which indicates the drastic decrease in crystallinity shown in Table III.

and 
Ultimate Goal of Young's Modulus of Simultaneous Biaxially Films

We shall discuss the ultimate value of Young's modulus of an ideal simultaneous biaxially stretched film whose crystallinity is $100 \%$ and the orientation of the $c$ axis to film surface is perfect. In this case, it is unnecessary to consider the composite mode of crystalline and amorphous phases and the mathematical treatment becomes simple. The relation between the intrinsic compliance of the structural unit and the bulk compliance is given by $^{42}$

$$
S_{i j k l}^{c v}=\sum_{r=1}^{3} \sum_{q=1}^{3} \sum_{p=1}^{3} \sum_{o=1}^{3}<a_{i o} a_{j p} a_{k q} a_{l r}>_{c v} S_{o p q r}^{c o}
$$

where $S_{i j k l}^{c v}$ are the bulk compliance and $S_{o p q r}^{c o}$ is their intrinsic compliance of a crystal unit. $a_{i o}$ is, for example, the direction cosine of the $u_{\mathrm{o}}$ axis with respect to the $X_{i}$ axis, which is given from the geometrical arrangements in Figure 10 as follows:

$$
a=\left|\begin{array}{cc}
\cos \phi \cos \theta \cos \eta-\sin \phi \sin \eta & \\
\sin \phi \cos \theta \cos \eta+\cos \phi \sin \eta & \\
-\sin \theta \cos \eta & \\
-\cos \phi \cos \theta \sin \eta-\sin \phi \cos \eta & \cos \phi \sin \theta \\
-\sin \phi \cos \theta \sin \eta+\cos \phi \cos \eta & \sin \phi \sin \theta \\
\sin \theta \sin \eta & \cos \theta
\end{array}\right|
$$

Average value of the crystal phase in eq 14, $<a_{i o} a_{j p} a_{k q}$ $a_{l r}>_{c v}$, is given by

$$
\begin{array}{r}
<a_{i o} a_{j p} a_{k q} a_{l r}>_{c v}=\int_{0}^{2 \pi} \int_{0}^{2 \pi} \int_{0}^{\pi} \omega(\theta, \eta) a_{i o} a_{j p} a_{k q} a_{l r} \\
\times \sin \theta \mathrm{d} \theta \mathrm{d} \phi \mathrm{d} \eta
\end{array}
$$

where $\omega(\theta, \eta)$ are an orientation distribution function of the crystal unit $0-U_{1} U_{2} U_{3}$ with respect to the coordinate $0-X_{1} X_{2} X_{3}$ in Figure 10 .

The generalized orientation factors $F_{l 0 n}$ of crystal phase can be represented by using $\omega(\theta, \eta)$ in the case when crystal units have an uniaxial orientation around the $X_{3}$ axis (film thickness direction):

$$
F_{l 0 n}=\int_{0}^{2 \pi} \int_{0}^{2 \pi} \int_{0}^{\pi} \omega(\theta, \eta) P_{l}^{n}(\cos \theta) \cos n \eta \sin \theta \mathrm{d} \theta \mathrm{d} \phi \mathrm{d} \eta
$$

The elastic compliance $S_{i j k l}$, represented as a tensor quantity, may be related to $S_{u v}$ by matrix as follows:

$$
\begin{aligned}
& S_{i j k l}=S_{u v} \quad u \text { and } v \leqq 3 \\
& S_{i j k l}=(1 / 2) \quad u \text { or } v \geqq 3 \\
& S_{i j k l}=(1 / 4) S_{u v} \quad u \text { and } v \geqq 3
\end{aligned}
$$

where $(i j)$ and $(k l)$ become $u$ and $v$, respectively. The combinations are as follows:

$$
(23) \rightarrow 4 \quad(31) \rightarrow 5 \quad(12) \rightarrow 6
$$

Using eqs $14-19$, the bulk compliance $S_{11}^{c v}\left(=S_{22}^{c v}\right)$ with $100 \%$ crystallinity in the stretching direction may be given by

$$
\begin{aligned}
& S_{11}^{c v}=S_{22}^{c v}=(1 / 64) S_{11}^{c o}\left\{(1 / 35) F_{404}-(8 / 35) F_{402}+(72 / 35) F_{400}\right. \\
& \left.-(32 / 7) F_{202}+(64 / 7) F_{200}+64 / 5\right\}+(1 / 64) S_{22}^{c o}\left\{(1 / 35) F_{404}\right. \\
& \left.+(8 / 35) F_{402}+(72 / 35) F_{400}+(32 / 7) F_{202}+(64 / 7) F_{200}+64 / 5\right\} \\
& +S_{33}^{c o}\left\{(3 / 35) F_{400}-(2 / 7) F_{200}+1 / 5\right\} \\
& +(1 / 64)\left(2 S_{12}^{c o}+S_{66}^{c o}\right)\left\{-(1 / 35) F_{404}+(24 / 35) F_{400}\right. \\
& \left.+(64 / 21) F_{200}+(64 / 15)\right\}+(1 / 16)\left(2 S_{13}^{c o}+S_{55}^{c o}\right)\left\{(2 / 35) F_{402}\right. \\
& \left.-(24 / 35) F_{400}-(4 / 21) F_{202}-(8 / 21) F_{200}+112 / 105\right\} \\
& +(1 / 16)\left(2 S_{23}^{c o}+S_{44}^{c o}\right)\left\{-(2 / 35) F_{402}-(24 / 35) F_{400}\right. \\
& \left.+(4 / 21)) F_{202}-(8 / 21) F_{200}+112 / 105\right\}
\end{aligned}
$$

where the orientation factors may be given by

$$
\begin{aligned}
& F_{200}=\frac{1}{2}\left(3<\cos ^{2} \theta>-1\right) \\
& F_{202}=3<\sin ^{2} \theta \cos 2 \eta> \\
& F_{400}=\frac{1}{8}\left(35<\cos ^{4} \theta>-30<\cos ^{2} \theta>+3\right) \\
& F_{402}=\frac{15}{2}<\left(7 \cos ^{2} \theta-1\right) \sin ^{2} \theta \cos 2 \eta> \\
& F_{404}=105<\sin ^{4} \theta \cos 4 \eta>
\end{aligned}
$$

The problem that now arises has been how the value of the intrinsic compliances $S_{u v}^{c o}$ of a polyethylene crystal unit can be determined theoretically. The values as the pioneering work were obtained by Odajima et al. ${ }^{43}$ and Tashiro et al. ${ }^{44}$ using a B matrix. However, their values of $S_{33}^{c o}$ are much higher than the observed values (213$235 \mathrm{GPa}$ ) by X-Ray diffraction, because of the lack of inharmonic effects, indicating that their theoretical values are useful only at very low temperature (close to the absolute zero point). Since then, the force fields have been improved considerably, and more recent calculations might compare much better with the experimental values. For example, Lacks and Rutledge estimated the crystal lattice modulus along the chain direction as 285 $\mathrm{GPa} .{ }^{45}$ They calculated elastic stiffness $C_{u v}^{c o}$ except $C_{44}^{c o}$, $C_{55}^{c o}$, and $C_{66}^{c o}$. Therefore, the elastic compliance $S_{u v}^{a o}$ needed in the numerical calculation cannot be obtained by using the inverse relationship. More recent investigation by Zehnder ${ }^{46}$ provided the best fit between the theoretical and measured values of crystal lattice modulus along the chain direction by X-Ray diffraction. ${ }^{7-8}$ Namely, his calculated modulus $\left(1 / S_{33}^{c o}\right)$ was $216 \mathrm{GPa}$, which is very close to our experimental value in the range $213-229 \mathrm{GPa}^{7}$ According to his paper, ${ }^{46} S_{u v}^{a o}$ is given by

$$
(11) \rightarrow 1(22) \rightarrow 2 \quad(33) \rightarrow 3
$$




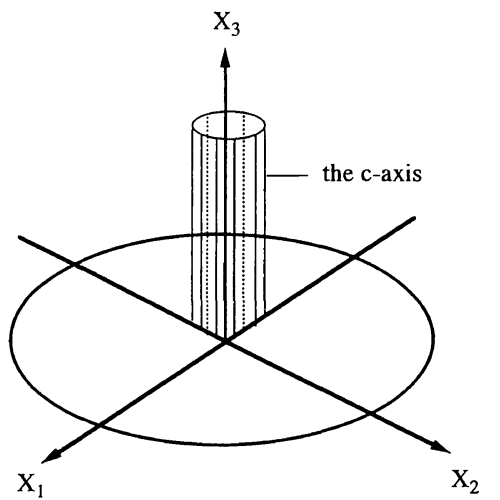

(a)

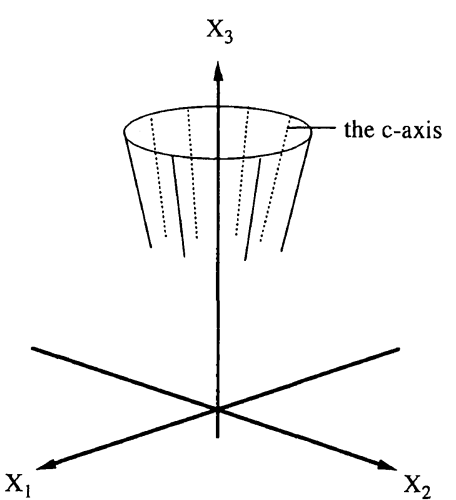

(b)

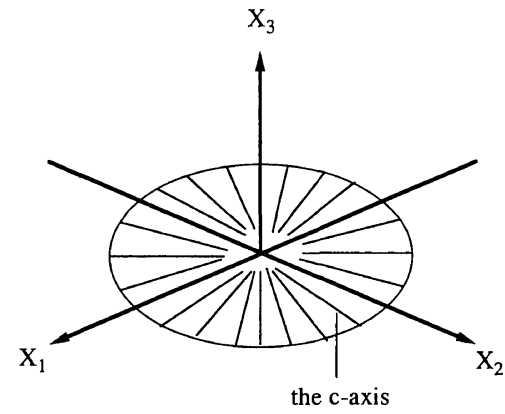

(c)

Figure 15. Orientational mode of the $c$-axes. (a) perfectly uniaxial orientation with respect to the stretching direction of the $X_{3}$ axis. (b) preferential orientation with respect to the stretching direction of the $X_{3}$ axis. (c) perfectly biaxial orientation with respect to the stretching direction of the $X_{1}$ and $X_{2}$ axes.

$$
S_{u v}^{c o}=\mid \begin{array}{cccc}
0.281 & -0.161 & -0.00036 & 0 \\
-0.172 & 0.258 & -0.0041 & 0 \\
-0.00088 & -0.0036 & 0.00462 & 0 \\
0 & 0 & 0 & 0.474 \\
0 & 0 & 0 & 0 \\
0 & 0 & 0 & 0
\end{array}
$$

$$
\begin{array}{cc|}
0 & 0 \\
0 & 0 \\
0 & 0 \\
0 & 0 \\
0.599 & 0 \\
0 & 0.431
\end{array} \mid \times 10^{-2} / \mathrm{GPa}
$$

The ultimate value of Young's modulus may be given as $1 / S_{11}^{c v}\left(=S_{22}^{c v}\right)$. In this case, two cases can be considered; 1$)$ the $c$-axis is oriented perfectly parallel to the film surface but the crystallites rotate randomly around their own $c$-axis. 2) the $c$-axis and the (200) plane are oriented perfectly parallel to the film surface. In former case, we obtain $F_{200}=-1 / 2, F_{400}=3 / 8, F_{202}=0, F_{402}=0$, and $F_{404}=0$, while in latter case, we obtain $F_{200}=-1 / 2$, $F_{400}=3 / 8, F_{202}=3, F_{402}=-15 / 2$, and $F_{404}=105$. In such as ideal case, it is evident that the mechanical property in bulk can be represented as infinitesimal deformation of an anisotropic elastic body using 36 independent crystal elastic compliances $S_{u v}^{c o}$ based on the homogeneous stress hypothesis.

Table V shows the results. The different values of Young's modulus are due to the different values of $S_{u v}^{c o}$ which are proposed by Zehnder, ${ }^{46}$ Odajima et al., ${ }^{43}$ and Tashiro et al. ${ }^{44}$ Anyway, the expected values are much lower than the ultimate value beyond $200 \mathrm{GPa}$ which is close to the crystal lattice modulus in the chain direction measured by X-Ray diffraction. To explain such a lower value of the biaxially stretched polyethylene film, schematic diagrams are proposed in Figure 15.

In Figure 15, model (a) shows a typical diagram representing the perfect orientation of the $c$-axes along the $X_{3}$ direction. The $X_{3}$ axis corresponds to the uniaxially stretching direction. This system assures the ultimate value of Young's modulus in bulk corresponding to the crystal lattice modulus along the $c$-axis. Model (b) shows the preferential orientation of the $c$-axes, which has
Table V. Theoretical calculation of Young's modulus of ideal biaxial stretching films, in which crystallinity is $100 \%$ and the $c$-axes are oriented parallel to the film surface perfectly

\begin{tabular}{lccc}
\hline & \multicolumn{3}{c}{ Young's modulus/GPa } \\
\cline { 2 - 4 } & Zehnder $^{\mathrm{a}}$ & Odajima $^{\mathrm{b}}$ & Tashiro $^{\mathrm{c}}$ \\
\hline Case I & 7.58 & 9.58 & 15.6 \\
Case II & 7.17 & 6.17 & 30.3 \\
\hline
\end{tabular}

Case I : Random orientation of crystallites around the $c$-axis.

Case II : Preferential orientation of the (200) plane parallel to the film surface.

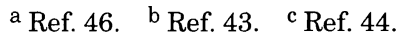

been usually observed for drawing of melt films. Model (c) shows the perfect orientation of the $c$-axes parallel to the film surface. The $X_{1}$ and $X_{2}$ axes correspond to the stretching directions. This system means that Young's modulus is isotropic on the film plane, which is quite different from the system shown in model (a). Accordingly, the ultimate value of Young's modulus of a simultaneous biaxially stretched film listed in Table $\mathrm{V}$ is less than 8 $\mathrm{GPa}$, if the crystal elastic compliance $S_{u v}^{c o}$ estimated by Zehnder $^{45}$ are realistic. This theoretical conclusion clearly supports the concept that high-modulus and high-strength polyethylene films cannot be produced by simultaneous biaxially stretching.

\section{CONCLUSION}

Simultaneous biaxially stretching was carried out by using UHMWPE dry gel prepared by crystallization from solutions. The maximum draw ratio depended on the concentration of solutions. The greatest significant drawability could be realized at $0.9 \mathrm{~g} / 100 \mathrm{~mL}$ with the maximum draw ratio $8.7 \times 8.7$, which is much higher than the optimum concentration of $0.45 \mathrm{~g} / 100 \mathrm{~mL}$ assuring the draw ratio $>300$-fold for uniaxial stretching. This indicates that one of the important factors to assure significant drawability under biaxially stretching is due to the existence of interpenetrating random coils which form a large number of coupling entanglements that are both intra- and intermolecular. The Young's modulus of the biaxially stretched film $(8.7 \times 8.7)$ were much lower than that of uniaxial film with almost same draw ratio. 
The crystallinity and melting point provided also the same tendency. Solid state ${ }^{13} \mathrm{C}$ NMR revealed that molecular chain mobility within the biaxially stretched films is more active than that within the uniaxially stretched films. The second order orientation factor of amorphous chain segments was estimated from birefringence by subtracting crystalline contribution from the total birefringence. These results indicate that the preferential orientation of the $c$-axis is mainly due to the rotation of crystallites around their $b$-axis leading to straining of tie molecules. Furthermore, the ultimate goal of Young's modulus was estimated by assuming an ideal simultaneous biaxially stretching film with $100 \%$ crystallinity and the perfect orientation of the $c$-axes parallel to the film surface. The theoretical values were less than $10 \mathrm{GPa}$, when the elastic compliance of a crystal unit by Odajima ${ }^{43}$ and Zehnder ${ }^{44}$ were employed.

\section{REFERENCES}

1. P. Smith, P. J. Lemstra, and B. Kalb, A. J. Pennings, Polym. Bull, 1, 733 (1979).

2. P. Smith and P. J. Lemstra, J. Mater. Sci., 15, 505 (1980),

3. P. Smith, P. J. Lemstra, J. P. L. Pippers, and A. M. Kiel, Colloid Polym. Sci., 258, 1070 (1981).

4. P. J. Smith and J. Lemstra, Polym. Sci., Polym. Phys. Ed., 19, 1007 (1981).

5. P. Smith, P. J. Lemstra, and H. C. Booij, J. Polym. Sci., Polym. Phys. Ed., 19, 877 (1981).

6. T. Ogita, R. Yamamoto, N. Suzuki, F. Ozaki, and M. Matsuo, Polymer, 32, 822 (1991).

7. M. Matsuo and C. Sawatari, Macromolecules, 19, 2036 (1986).

8. I. Sakurada, Y. Nukushina, and T. Ito, J. Polym. Sci., 57, 651 (1962).

9. S. Kavesh and D. C. Prevorsek, Int. J. Polym. Mater., 30, 15 (1995).

10. N. Sakaguchi, T. Oda, A. Nakai, and H. Kawai, Sen-i Gakkaishi, 33, 499 (1977).

11. H. Takahara, H. Kawai, Y. Yamaguchi, and A. Fukushima, Sen-i Gakkaishi, 25, 60 (1969).

12. S. Hibi, M. Maeda, M. Mizuno, S. Nomura, and H. Kawai, Sen-i Gakkaishi, 29, 152 (1973).

13. Y. Sakai and K. Miyasaka, Polymer, 29, 1608 (1988).

14. Y. Sakai and K. Miyasaka, Polymer, 31, 51 (1990).

15. Y. Sakai, K. Uematsu, and K. Miyasaka, Polymer, 34, 318 (1993).

16. N. S. J. A. Gerrits, R. J. Young, and L. P. J. Lemsstra, Polymer, 31, 231 (1990).

17. N. S. J. A. Gerrits and P. J. Lemsstra, Polymer, 32, 1770
(1991).

18. N. S. J. A. Gerrits and R. J. Young, J. Polym. Sci., Part B: Polym. Phys., 29, 825 (1991).

19. C. W. Bastiaansen, P. J. R. Leblands, and P. Smith, Macromolecules, 23, 2365 (1990).

20. C. Sawatari and M. Matsuo, Macromolecules, 19, 2726 (1986).

21. M. Matsuo, Macromolecules, 23, 3261 (1990).

22. R. J. Roe and W. R. Krigbaum, J. Chem. Phys., 40, 2608 (1964).

23. W. R. Krigbaum and R. J. Roe, J. Chem. Phys., 41, 737 (1964).

24. R. J. Roe, J. Appl. Phys., 36, 2024 (1965).

25. C. W. Bunn, Trans. Faraday Soc., 35, 482 (1939),

26. C. Sawatari and M. Matsuo, Colloid Polym. Sci., 263, 783 (1985).

27. M. Matsuo, C. Sawatari, and T. Ohhata, Macromolecules, 21, 1317 (1988)

28. C. Sawatari, S. Shimigiri, and M. Matsuo, Macromolecules, 20, 1033 (1987)

29. M. Matsuo, K. Inoue, and N. Abumiya, Sen-i Gakkaishi, 32 841 (1983).

30. M. Takayanagi and M. Matsuo, J. Macromol. Sci., Phys., B1, 407 (1967).

31. R. A. V. Raff, "In Encyclopedia of Polymer Science and Technology"; Marh, H.F. et al., Ed., New York, N.Y. 1967, 6, pp $275-332$.

32. Z. Hosemann, Phys., 127, 16 (1949).

33. D. J. Blundell, Acta Crysallogr., A26, 472 (1970).

34. M. Matsuo, C. Sawatari, M. Tsuji, and R. S. T. Manley, J. Chem. Soc., Faraday Trans. 2, 79, 1593 (1983).

35. M. Matsuo and C. Kitayama, Polym. J., 17, 479 (1985).

36. T. Hashimoto, M. Shibayama, and H. Kawai, Macromolecules, 131237 (1980).

37. C. Sawatari, T. Okumura, and M. Matsuo, Polym. J., 18, 741 (1986).

38. M. Matsuo, C. Sawatari, M. Iida, and M. Yoneda, Polym. J., 17, 1197 (1985)

39. W. Spendly, G. R. Hext, and F. R. Himsworth, Technometrics, 4, 441 (1962).

40. R. S. Stein and F. H. Norris, J. Polym. Sci., 21, 381 (1956).

41. S. Hoshino, J. Powers, D. G. Legrand, H. Kawai, and R. S. Stein, J. Polym. Sci., 58, 185 (1962).

42. V. B. Gupta and I. M. Ward, J. Macromol. Sci., B1, 373 (1967).

43. S. Odajima and T. Maeda, J. Polym. Sci. Part C, 15, 55 (1966).

44. K. Tashiro, M. Kobayashi, and H. Tadokoro, Macromolecules, 11, 914 (1978).

45. D. J. Lacks and G. C. Rutledge, J. Phy. Chem., 98, 1222 (1994).

46. M. Zehnder, Ph. D. Thesis "Atomic Simulation of the Elasticity of Polymers", ETH-Zurich 1997. 